\title{
AN IDENTIFICATION THEOREM FOR GROUPS WITH SOCLE PSU $_{6}(2)$
}

\author{
CHRIS PARKER ${ }^{\bowtie}$ and GERNOT STROTH
}

(Received 27 February 2011; accepted 10 February 2012; first published online 25 February 2013)

\author{
Communicated by E. A. O’Brien
}

\begin{abstract}
We identify the groups $\operatorname{PSU}_{6}(2), \operatorname{PSU}_{6}(2): 2, \operatorname{PSU}_{6}(2): 3$ and $\operatorname{Aut}\left(\operatorname{PSU}_{6}(2)\right)$ from the structure of the centralizer of an element of order three.

2010 Mathematics subject classification: 20 D05.

Keywords and phrases: groups, simple groups, identification of simple groups, classical groups, classification of finite simple groups.
\end{abstract}

\section{Introduction}

The aim of this paper is to provide a 3-local identification of the groups $\operatorname{PSU}_{6}(2): 3$, $\mathrm{PSU}_{6}(2): 2$ and $\mathrm{PSU}_{6}(2): \operatorname{Sym}(3)$. The results will be applied in a larger project which began in [12] and aims to provide an alternative proof for some major parts of the classification of the finite simple groups. The group $\mathrm{PSU}_{6}(2)$ is a Lie type group defined in characteristic 2 and so its appearance in a setting where 3 is the significant prime is unusual. This cross-characteristic behaviour is in part caused by the fact that the smaller group $\mathrm{SU}_{3}(2)$ is soluble and contains a normal subgroup of order 27 . In $\mathrm{SU}_{6}(2)$ there is a direct product $\mathrm{SU}_{3}(2) \times \mathrm{SU}_{3}(2)$ which projects in $X=\mathrm{PSU}_{6}(2)$ to a group which has normal subgroup $Q$ of order $3^{5}=243$. The subgroup $Q$ is an example of a large 3-subgroup of $X$ and and its normalizer is an example of a 3-local subgroup of $X$. We recall that for a prime $p$, a $p$-local subgroup of a group $G$ is by definition the normalizer in $G$ of a nontrivial $p$-subgroup of $G$. We shall say more about large $p$-subgroups in a moment.

For subgroups $X \leq Y \leq G$, we say that $X$ is weakly closed in $Y$ with respect to $G$ provided that if $g \in G$ and $X^{g} \leq Y$, then $X^{g}=X$. A group $X$ is said to be quasisimple provided $X / Z(X)$ is a simple group and $X=[X, X]$. A component of $G$ is a subnormal quasisimple subgroup of $G$. The fitting subgroup of $G, F(G)$, is the largest normal

(c) 2013 Australian Mathematical Publishing Association Inc. 1446-7887/2013 \$16.00 
nilpotent subgroup of $G$ and the layer of $G, E(G)$, is the subgroup of $G$ which is generated by all the components of $G$. The generalized fitting subgroup of $G, F^{*}(G)$, is defined by $F^{*}(G)=F(G) E(G)$. See [1, Ch. 11] for the fundamental properties of this characteristic subgroup of $G$, the most important property being that $C_{G}\left(F^{*}(G)\right) \leq$ $F^{*}(G)$. We say that a $p$-subgroup $Q$ of a group $G$ is large provided:

(L1) $F^{*}\left(N_{G}(Q)\right)=Q$; and

(L2) if $U$ is a nontrivial subgroup of $Z(Q)$, then $N_{G}(U) \leq N_{G}(Q)$.

An interesting observation is that most of the groups of Lie type in characteristic $p$ contain a large $p$-subgroup. In fact the only Lie type groups in characteristic $p$ and rank at least 2 which do not contain such a subgroup are $\operatorname{PSp}_{2 n}\left(2^{a}\right), \mathrm{F}_{4}\left(2^{a}\right)$ and $\mathrm{G}_{2}\left(3^{a}\right)$. It is not difficult to show that groups $G$ which contain a large $p$-subgroup are of parabolic characteristic $p$, which means that all $p$-local overgroups $N$ of a Sylow $p$-subgroup $S$ satisfy $F^{*}(N)=O_{p}(N)$ [17, Lemma 2.1]. The work in [12] initiates the determination of the $p$-local overgroups of $S$ which are not contained in $N_{G}(Q)$. This is the first mile of a long road to showing that typically a group with a large $p$-subgroup is a group of Lie type defined in characteristic $p$ and of rank at least 2 . The basic idea is to gather information about the $p$-local subgroups of $G$ containing a fixed Sylow $p$ subgroup so that the subgroup generated by them can be identified with a group of Lie type via its action on the chamber complex coming from these subgroups (which will in fact be the maximal parabolic subgroups). However, one is sometimes confronted with the following situation. Some (but perhaps not all) of the $p$-local subgroups of $G$ containing a given Sylow $p$-subgroup $S$ of $G$ generate a subgroup $H$ and $F^{*}(H)$ is known to be isomorphic to a Lie type group in characteristic $p$. The expectation (or rather hope) is that $G=H$. Under the assumption that $H$ is a proper subgroup of $G$, one usually tries to prove that $H$ contains all the $p$-local subgroups of $G$ which contain $S$ and then in a next step to prove that $H$ is strongly $p$-embedded in $G$, at which stage [16] is applicable and delivers the contradiction $G=H$. The last two steps are reasonably well understood, at least for groups with mild extra assumptions imposed. However, it might be that the first step cannot be made. Typically this will occur only when $N_{G}(Q)$ is not contained in $H$. If $N_{H}(Q)$ is nonsoluble and $p$ is odd, Seidel has shown in his PhD thesis [22] that this cannot occur. In [17] the authors use the identification theorem presented in this paper together with further identifications [14, 15, 18-20] to handle the more delicate analysis when $p=3$ and $N_{H}(Q)$ is soluble. Far from these configurations not arising, the rule of thumb in these cases is that if it might happen then it does. The possibilities for the group $F^{*}(H)$ are easily shown to be $\operatorname{PSL}_{3}\left(3^{a}\right)$ (which we do not consider), $\mathrm{PSp}_{4}(3), \mathrm{PSU}_{4}(3), \mathrm{PSL}_{4}(3), \Omega_{7}(3), \mathrm{P}_{8}^{+}(3)$ and $\mathrm{G}_{2}\left(3^{a}\right)$. The main theorem in [17] says that if $N_{G}(Q) \not \leq H$, then $F^{*}(G)$ is one of $\mathrm{U}_{6}(2), \mathrm{F}_{4}(2)$, ${ }^{2} \mathrm{E}_{6}(2), \mathrm{McL}, \mathrm{Co}_{2}, \mathrm{M}(22), \mathrm{M}(23)$ or $\mathrm{F}_{2}$, where $\mathrm{M}(22)$ and $\mathrm{M}(23)$ are the Fischer groups which are alternatively denoted by $\mathrm{Fi}_{22}$ and $\mathrm{Fi}_{23}$, and $\mathrm{F}_{2}$ represents the Baby monster sporadic simple group.

Thus the present paper is required for the identification of groups $G$ with $F^{*}(G) \cong$ $\mathrm{U}_{6}(2)$ in the case where $F^{*}(H) \cong \mathrm{U}_{4}(3)$. Furthermore, this paper plays a pivotal role in 
the 3-local identifications of $\mathrm{M}(22)$ and ${ }^{2} \mathrm{E}_{6}(2)$. Indeed, the centralizers of involutions in both $\mathrm{M}(22)$ and ${ }^{2} \mathrm{E}_{6}(2)$ and their automorphism groups prominently feature groups with socle $\mathrm{U}_{6}(2)$. In addition, these identifications are required for our work on the sporadic simple groups $\mathrm{M}(23)$ and $\mathrm{F}_{2}$.

In earlier work [13] the first author proved the following result. Let $G$ be a finite group, $S$ be a Sylow 3-subgroup of $G$ and $Z=Z(S)$. Assume that $N_{G}(Z)$ is similar to a 3-normalizer in $\mathrm{PSU}_{6}(2)$ (see [13]). Then either $G \cong \mathrm{PSU}_{6}(2)$ or $Z$ is weakly closed in $S$. However, for our intended applications of such results as outlined above, we also need to identify the groups $\operatorname{PSU}_{6}(2): 3, \operatorname{PSU}_{6}(2): 2$ and $\operatorname{PSU}_{6}(2): \operatorname{Sym}(3)$ from their 3-local data (here and throughout this work we use the Atlas [3] notation for group extensions). The addition of these automorphisms causes numerous difficulties.

Definition 1.1. We say that $X$ is similar to a 3-centralizer in a group of type $\operatorname{PSU}_{6}(2)$ or $\mathrm{F}_{4}(2)$ provided the following conditions hold:

(i) $Q=F^{*}(X)$ is extraspecial of order $3^{5}$ and $Z\left(F^{*}(X)\right)=Z(X)$; and

(ii) $X / Q$ contains a normal subgroup isomorphic to $\mathrm{Q}_{8} \times \mathrm{Q}_{8}$.

Thus when we say that $X$ is similar to a 3-centralizer in a group of type $\operatorname{PSU}_{6}(2)$ or $\mathrm{F}_{4}(2)$ we mean that $X$ has approximately the same structure as the centralizer in $\mathrm{PSU}_{6}(2)$ or $\mathrm{F}_{4}(2)$ of a certain element of order three. A precise description of the possibilities for the group $X / Q$ will be determined in Section 3. Our theorem is as follows.

Theorem 1.2. Suppose that $G$ is a group, $Z \leq G$ has order three and set $H=C_{G}(Z)$. If $\mathrm{H}$ is similar to a 3-centralizer in a group of type $\mathrm{PSU}_{6}(2)$ or $\mathrm{F}_{4}(2)$ and $Z$ is weakly closed in $F^{*}(H)$ but not in $H$ with respect to $G$, then $G \cong \operatorname{PSU}_{6}(2)$, $\operatorname{PSU}_{6}(2): 2$, $\mathrm{PSU}_{6}(2): 3$ or $\mathrm{PSU}_{6}(2): \operatorname{Sym}(3)$.

In the case where $Z$ is weakly closed in $H, G$ could be a nilpotent group extended by a group similar to a 3-centralizer of type $\operatorname{PSU}_{6}(2)$ or $\mathrm{F}_{4}(2)$. Thus the hypothesis that $Z$ is not weakly closed in $H$ is necessary in order to have an identification theorem. On the other hand, the hypothesis that $Z$ is weakly closed in $F^{*}(H)$ is needed to prevent further examples related to $\mathrm{F}_{4}(2)$ arising. The methods we use in this paper are also applicable to the configuration arising in $\mathrm{F}_{4}(2)$. However the investigation of this possibility takes a rather different road at the very outset of our proof and so the analysis of this case is not included here but is the subject of [18]. Combining the work of both papers, we obtain the following theorem.

Theorem 1.3. Suppose that $G$ is a group, $Z \leq G$ has order three and set $H=C_{G}(Z)$. If $H$ is similar to a 3-centralizer in a group of type $\mathrm{PSU}_{6}(2)$ or $\mathrm{F}_{4}(2)$ and $\mathrm{Z}$ is not weakly closed in a Sylow 3-subgroup of $G$ with respect to $G$, then either $F^{*}(G) \cong \mathrm{F}_{4}(2)$ or $F^{*}(G) \cong \mathrm{PSU}_{6}(2)$.

We now describe the layout of the paper and highlight a number of interesting features. We begin in Section 2 with preliminary lemmas and background material. Noteworthy results in this section are Lemma 2.5, where we embellish the statement 
of Hayden's theorem [9] to give the structure of the normal subgroup of index 3, and Lemma 2.13, where we use transfer theorems to show that a group with a certain specified 2-local subgroup has a subgroup of index 2. The relevance of such results to our proof is apparent as the list of groups in the conclusion of our theorem shows. Let $G, H$ and $Z$ be as in the statement of Theorem 1.2 and let $S \in \operatorname{Syl}_{3}(M)$ where $M=N_{G}(Z)$ contains $H$ of index at most 2. In Section 3 we tease out the structure of $M$ and establish much of the notation that is used in the proof of Theorem 1.2.

In Section 4 we determine the structure of the normalizer of another 3-subgroup which we call $J$. The fact that $N_{G}(J)$ is not contained in $M$ is a consequence of the hypothesis that $Z$ is not weakly closed in $M$. We find in Lemma 4.5 that $N_{G}(J) / J \cong$ $2 \times \operatorname{Sym}(6)$ or $\operatorname{Sym}(6)$. With this information, after using a transfer theorem, we are able to apply [13], and do so in Theorem 4.7 to get that $G \cong \operatorname{PSU}_{6}(2)$ or $\mathrm{PSU}_{6}(2): 3$ if $N_{M}(S) / S \cong \operatorname{Dih}(8)$. Thus from this stage on we assume that $N_{M}(S) / S \cong 2 \times \operatorname{Dih}(8)$ and $N_{M}(J) / J \cong 2 \times \operatorname{Sym}(6)$. With this assumption, our target groups all have a subgroup of index 2. Our plan is to determine the structure of a 2-central involution $r$, apply Lemma 2.13 and then apply Theorem 4.7 to the subgroup of index 2. The involution we focus on is contained in $M$ and centralizes a subgroup of $F^{*}(M)$ isomorphic to $3_{+}^{1+2}$. But before we can make this investigation we need to determine the centralizer of another subgroup (for now we will call it $X$ ) which has order either three or nine. It turns out that we may apply the theorems of Hayden [9] and Prince [21] to get $E\left(C_{G}(X)\right) \cong \mathrm{SU}_{4}(2)$. At this juncture, given the 3-local information that we have gathered, we can construct an extraspecial 2-subgroup $\Sigma$ of order $2^{9}$ in $K=C_{G}(r)$. In Theorem 5.5 we show that $N_{K}(\Sigma) / \Sigma \cong \operatorname{Aut}\left(\mathrm{SU}_{4}(2)\right),\left(\mathrm{SU}_{4}(2) \times 3\right): 2$ or $\mathrm{Sp}_{6}(2)$. In our target groups the possibility $\mathrm{Sp}_{6}(2)$ does not arise; we will say more about this shortly.

In Section 6 we show that $\Sigma$ is strongly closed in $N_{K}(\Sigma)$ with respect to $K$ and then we apply Goldschmidt's theorem to get that $K=N_{K}(\Sigma)$. At this stage we know the centralizer of a 2-central involution in $G$ and so we can finally prove Theorem 1.2 in Section 7. When $K / \Sigma \cong \operatorname{Sp}_{6}(2)$ we apply [23] to obtain $G \cong \mathrm{Co}_{2}$ and then eliminate this group as it does not satisfy our hypothesis on the structure of $M$. One wonders if the configuration involving $\mathrm{Sp}_{6}(2)$ could be eliminated at an earlier stage. However, as $\mathrm{Co}_{2}$ contains $\mathrm{PSU}_{6}(2): 2$ as a subgroup of index 2300, these groups are intimately related. A 3-local identification of $\mathrm{Co}_{2}$ can be found in [15].

Our notation follows that of $[1,6,7]$. In particular, we use the definition of signalizers as given in [7, Definition 23.1] as well as the notation $И_{G}(A, \pi)$ to denote the set of $A$-signalizers in $G$. As mentioned earlier, we use the Atlas [3] notation for group extensions. We also use [3] as a convenient source for information about subgroups of almost simple groups. Often this information can be easily gleaned from well-known properties of classical groups. For odd $p$, the extraspecial groups of exponent $p$ and order $p^{2 n+1}$ are denoted by $p_{+}^{1+2 n}$. The extraspecial 2-groups of order $2^{2 n+1}$ are denoted by $2_{+}^{1+2 n}$ if the maximal elementary abelian subgroups have order $2^{1+n}$, otherwise we write $2_{-}^{1+2 n}$. Our notation for specific groups is self-explanatory. For a subset $X$ of a group $G, X^{G}$ denotes the set of $G$-conjugates of $X$. If $x, y \in H \leq G$, 
we often write $x \sim_{H} y$ to indicate that $x$ and $y$ are conjugate in $H$. All the groups in this paper are finite groups.

\section{Preliminary results}

In this section we gather together preliminary results for our proof of Theorem 1.2. For a group $G$ with Sylow $p$-subgroup $P$ and $v \in P, v$ is extremal in $P$ if $C_{P}(v)$ is a Sylow $p$-subgroup of $C_{G}(v)$.

Lemma 2.1. Suppose that $p$ is a prime and $G$ is a group. Let $P$ be a Sylow p-subgroup of $G$ and let $Q$ be a proper normal subgroup of $P$ such that $P / Q$ is cyclic. Assume that there is $u \in P \backslash Q$ such that:

(a) no conjugate of $u^{p}$ is contained in $P \backslash Q$; and

(b) any extremal conjugate of $u$ in $P$ is contained in $Q \cup Q u$.

Then either $G$ has a normal subgroup $N$ with $G / N$ cyclic and $u \notin N$ or there is $g \in G$ such that:

(i) $u^{g} \in Q$;

(ii) $u^{g}$ is extremal in $P$; and

(iii) $C_{P}(u)^{g} \leq C_{P}\left(u^{g}\right)$.

Proof. See [7, Proposition 15.15] or [24, Corollary 5.3.1].

Lemma 2.2. Suppose that $p$ is a prime, $G$ is a group and $P \in \operatorname{Syl}_{p}(G)$.

(i) Assume that there is a normal subgroup $Q$ of $P$ such that $P / Q$ is cyclic and that $y \in P \backslash Q$ has order $p$. If every extremal conjugate of $y$ in $P$ is contained in $Q y$, then $G$ has a normal subgroup $N$ with $y \notin N$ and $G / N$ cyclic.

(ii) Assume that $P \leq M \leq G, y \in P \backslash M^{\prime}$ has order $p$ and that, if $x \in G$ with $y^{x} \in P$ extremal, then there is $g \in M$ such that $y^{x}=y^{g}$. Then $y \notin G^{\prime}$.

(iii) Assume that $J=J(P)$ is the Thompson subgroup of $P$. If $J$ is elementary abelian and $J \not N_{G}(J)^{\prime}$, then $J \not \leq G^{\prime}$.

Proof. (i) This follows from Lemma 2.1.

(ii) As $M / M^{\prime}$ is abelian, there is $N \leq M$ such that $M^{\prime} \leq N, y \notin N, M=N P$ and $P /(P \cap N)$ is cyclic. Set $Q=P \cap N$. Now for $g \in M$ with $y^{g} \in P, y^{g} \in Q y$. Hence by assumption $y^{x} \in Q y$ for all $x \in G$ such that $y^{x}$ is extremal in $P$. Now (ii) follows from (i).

(iii) Set $M=N_{G}(J)$ and pick $y \in J \backslash M^{\prime}$. Assume that $g \in G$ and $y^{g}$ is extremal in $P$. Then $C_{P}\left(y^{g}\right) \in \operatorname{Syl}_{p}\left(C_{G}\left(y^{g}\right)\right)$. Since $C_{G}(y)$ contains $J$, it follows that $C_{P}\left(y^{g}\right)$ contains a $G$-conjugate of $J$. Since $J$ is weakly closed in $P$, we have $J \leq C_{P}\left(y^{g}\right)$. But then $y^{g} \in C_{P}(J) \leq J$. Since $M$ controls fusion in $J$, we now have that $y^{g}=y^{m}$ for some $m \in M$. Now (iii) follows from (ii).

Lemma 2.3. Suppose that $F$ is a field, $V$ is an n-dimensional vector space over $F$ and $G=\mathrm{GL}(V)$. Assume that $q$ is quadratic form of Witt index at least 1 and $\mathcal{S}$ is the set of 
singular one-dimensional subspaces of $V$ with respect to $q$. Then the stabilizer in $G$ of $\mathcal{S}$ preserves q up to similarity.

Proof. See [15, Lemma 2.10].

Lemma 2.4. Suppose that $p$ is an odd prime, $X=\mathrm{GL}_{4}(p)$ and $V$ is the natural $\mathrm{GF}(p) X$ module. Let $A=\langle a, b\rangle \leq X$ be elementary abelian of order $p^{2}$ and assume that $[V, a]=C_{V}(b)$ and $[V, b]=C_{V}(a)$ are distinct and of dimension two. Let $v \in V \backslash[V, A]$. Then A leaves invariant a nondegenerate quadratic form with respect to which $v$ is a singular vector. In particular, $X$ contains exactly two conjugacy classes of subgroups such as $A$. One is conjugate to a Sylow p-subgroup of $\mathrm{GO}_{4}^{+}(p)$ and the other to a Sylow p-subgroup of $\mathrm{GO}_{4}^{-}(p)$.

Proof. See [15, Lemma 2.11].

LeMma 2.5. Let $H$ be a finite group and let $d \in H$ have order three such that $X=C_{H}(d)$ is isomorphic to the centralizer of a nontrivial 3-central element in $\operatorname{PSp}_{4}(3)$. Let $P \in \operatorname{Syl}_{3}(X)$ and $E$ be the elementary abelian subgroup of $P$ of order 27. Assume that $E$ does not normalize any nontrivial 3 -subgroup of $H$, that $d$ is not $H$-conjugate to its inverse, and $H$ has a normal subgroup of index 3. Then $H=C_{H}(d)$.

Proof. Notice first of all that $P \in \operatorname{Syl}_{3}(H)$. Let $H_{1}$ be a normal subgroup of $H$ of index 3 and set $E_{1}=E \cap H$. So $C_{H_{1}}(d) \cong 3_{+}^{1+2}: \mathrm{Q}_{8}$ and $E_{1}$ has order nine. Suppose that $x \in E_{1} \backslash\langle d\rangle$. We see that all subgroups of order three in $E_{1}$ different from $\langle d\rangle$ are conjugate in $O_{3}\left(C_{H}(d)\right)$ and so every element of $E_{1} \backslash\langle d\rangle$ is conjugate to its inverse. As $d$ is not conjugate to its inverse, $d$ is the unique conjugate of $d$ in $E_{1}$. Furthermore, $d$ is not conjugate to any element of $E \backslash H^{\prime}$ and so $d$ is the unique conjugate of $d$ in $E$. Since $x$ is not conjugate to $d$, we have that $E_{1}=\langle d, x\rangle$ is a Sylow 3-subgroup of $C_{H_{1}}(x)$. As $E_{1} /\langle x\rangle$ is self-normalizing in $C_{H_{1}}(x) /\langle x\rangle, C_{H_{1}}(x)$ has a normal 3-complement $T$ by Burnside's theorem. However, $C_{H_{1}}(x)$ is normalized by $E$ and so $T=1$ by hypothesis. It follows that $C_{H}(x)=E$ for all $x \in E_{1} \backslash\langle d\rangle$.

Let $y \in E \backslash H_{1}$. Then, as before, $E_{1}$ is a Sylow 3-subgroup of $C_{H_{1}}(y)$. Since $d$ is not conjugate to any nontrivial element of $E_{1} \backslash\{d\}$, we have $N_{H}\left(E_{1}\right) \leq X$. So $N_{C_{H_{1}}(y)}\left(E_{1}\right)=\left\langle E_{1}, s\right\rangle$ where $s$ is an element of order at most two in $X$. Since $\left[E_{1}, s\right]<$ $E_{1}$, Grün's theorem [6, Ch. 7, Theorem 4.4] implies that $C_{H_{1}}(y)$ has a subgroup $L$ of index at least $\left|E_{1}:\left[E_{1}, s\right]\right|$ with Sylow 3-subgroup $\left[E_{1}, s\right]$. Since $L$ is normalized by $E$, we also have $O_{3^{\prime}}(L)=1$. Hence, if $s=1$, then $C_{H}(y) \leq X$ which means that $C_{H}(y)=E$. So suppose that $\left[E_{1}, s\right]$ has order three. Then, as $C_{H}\left(\left[E_{1}, s\right]\right)=E$, we have $\left[E_{1}, s\right]$ is self-centralizing in $L$. Applying the other Feit-Thompson theorem [4] to $L$ and using $O_{3^{\prime}}(L)=1$, we now have that either $L \cong \operatorname{Sym}(3)$ with $L=N_{X \cap H_{1}}\left(\left[E_{1}, s\right]\right)$ or $L \cong \mathrm{PSL}_{3}(2)$ or $\operatorname{Alt}(5)$. The latter two cases are eliminated as $L$ is normalized by $E_{1}$ and the centralizers of all of the nontrivial elements of $E_{1}$ are soluble. Therefore, $C_{H}(y)=C_{X}(y) \leq X$ for all $y \in E \backslash E_{1}$. 
Now let $R \in \operatorname{Syl}_{2}(X)$ and $r \in R$ be an involution. Then $C_{X}(r)=R\langle d, y\rangle$ for some $y \in E \backslash E_{1}$. Furthermore, as $d$ is the unique conjugate of $d \in\langle d, y\rangle$,

$$
N_{C_{H}(r)}(\langle d, y\rangle)=N_{X}(\langle d, y, r\rangle)=\langle d, y, r\rangle
$$

and so $C_{H}(r)$ has a normal 3-complement $U$ by Burnside's theorem. Finally,

$$
U=\left\langle C_{U}(w) \mid w \in\langle d, y\rangle^{\#}\right\rangle \leq X
$$

as $C_{H}(w) \leq X$ for each $w \in\langle d, y\rangle^{\#}$. It follows that $U=R$. But then $R \in \operatorname{Syl}_{2}(H)$ and $r \in Z^{*}(H)$ by [2]. As $\left[O_{3}(X), r\right]=O_{3}(X)$, we conclude that $O_{3}(X) \leq O_{2^{\prime}}(H)$ and deduce that $H=X$ from the Frattini argument. This completes the proof of the lemma.

THeOREm 2.6 (Hayden). Let $H$ be a finite group and let $d$ be an element of order three in $H$ such that $X=C_{H}(d)$ is isomorphic to the centralizer of a nontrivial 3-central element in $\mathrm{PSp}_{4}(3)$. Let $P \in \operatorname{Syl}_{3}(X)$ and $E$ be the elementary abelian subgroup of $P$ of order 27. If $E$ does not normalize any nontrivial $3^{\prime}$-subgroup of $H$ and $d$ is not $H$-conjugate to its inverse, then either $H=X$ or $H \cong \mathrm{PSp}_{4}(3)$.

Proof. By [9] either $H \cong \mathrm{PSp}_{4}$ (3) or $H$ has a normal subgroup of index 3 . The result now follows from Lemma 2.5.

Theorem 2.7 (Prince). Let $H$ be a finite group and let $d$ be an element of order three in $H$ such that $X=C_{H}(d)$ is isomorphic to the centralizer of a nontrivial 3-central element in $\mathrm{PSp}_{4}(3)$. Let $P \in \operatorname{Syl}_{3}\left(C_{H}(d)\right)$ and $E$ be the elementary abelian subgroup of $P$ of order 27. If $E$ does not normalize any nontrivial $3^{\prime}$-subgroup of $H$ and $d$ is $H$-conjugate to its inverse, then one of the following properties holds:

(i) $\left|H: C_{H}(d)\right|=2$;

(ii) $H$ is isomorphic to $\operatorname{Aut}\left(\mathrm{SU}_{4}(2)\right)$; or

(iii) $H$ is isomorphic to $\mathrm{Sp}_{6}(2)$.

Proof. See [21, Theorem 2].

Lemma 2.8. Suppose that $X$ is a group of shape $3_{+}^{1+2} \cdot \mathrm{SL}_{2}(3), O_{2}(X)=1$ and a Sylow 3 -subgroup of $X$ contains an elementary abelian subgroup of order $3^{3}$. Then $X$ is isomorphic to the centralizer of a nontrivial 3-central element in $\mathrm{PSp}_{4}(3)$.

Proof. See [13, Lemma 6].

Lemma 2.9. Let $G$ be a finite group and $S$ be a Sylow 3-subgroup of $G$. Set $Z=Z(S)$, $M=N_{G}(Z)$ and let $J=J(S)$ be the Thompson subgroup of $S$. Suppose that $G^{*}$ is a normal subgroup of $G$ and set $M^{*}=M \cap G^{*}$. Assume that the following properties hold:

(i) $\left|M^{*}\right|=2^{7} \cdot 3^{6}$;

(ii) $M^{*} \geq Q R=O_{3,2}\left(M^{*}\right)$, where $Q=O_{3}\left(M^{*}\right)$ is extraspecial of order $3^{5}$ and $R \in$ $\operatorname{Syl}_{2}\left(O_{3,2}\left(M^{*}\right)\right)$;

(iii) $O^{2}\left(M^{*}\right)=\left(S \cap M^{*}\right) R$ has index 2 in $M^{*}$; and

(iv) $Q / Z$ is an $M^{*}$-chieffactor. 
TABLE 1. Involutions in $\mathrm{Sp}_{6}(2)$ and $\operatorname{Aut}\left(\mathrm{SU}_{4}(2)\right)$.

\begin{tabular}{cccc}
\hline & $\operatorname{Aut}\left(\mathrm{SU}_{4}(2)\right)$ & $\operatorname{Sp}_{6}(2)$ & $\operatorname{dim} C_{V}\left(u_{j}\right)$ \\
\hline$u_{1}$ & $2_{+}^{1+4} \cdot(\operatorname{Sym}(3) \times \operatorname{Sym}(3))$ & $2^{7} \cdot(\operatorname{Sym}(3) \times \operatorname{Sym}(3))$ & 6 \\
$u_{2}$ & $2^{6} .3$ & $2^{7} .3$ & 4 \\
$u_{3}$ & $2 \times \operatorname{Sym}(6)$ & $2^{5} \cdot \operatorname{Sym}(6)$ & 4 \\
$u_{4}$ & $2 \times(\operatorname{Sym}(4) \times 2)$ & $2^{9} .3$ & 4 \\
\hline
\end{tabular}

If $N_{G^{*}}\left(J \cap G^{*}\right) \not \leq M^{*}$, then $G^{*} \cong \mathrm{PSU}_{6}(2)$ and $G$ is a subgroup of $\operatorname{Aut}\left(\mathrm{PSU}_{6}(2)\right)$ such that $G / G^{*} \cong M / M^{*}$.

Proof. Since $N_{G^{*}}\left(J \cap G^{*}\right) \not \leq M^{*}, Z$ is not weakly closed in $S \cap G^{*}$. The conditions imposed on the structure of $M^{*}$ imply that $M^{*}$ is similar to a 3-normalizer in $\operatorname{PSU}_{6}(2)$ [13, Definition 1]. Hence [13, Theorem 1] gives the result.

Lemma 2.10. Suppose that $E$ is an extraspecial 2-group and $x \in \operatorname{Aut}(E)$ is an involution. If $C_{E}(x) \geq[E, x]$, then $[E, x]$ is elementary abelian.

Proof. Let $\langle e\rangle=Z(E)$. We show that every element of $[E, x]$ has order two. Let $f \in[E, x] \backslash\langle e\rangle$. Then $f e$ has the same order as $f$. Thus we may suppose that $f=[h, x]$ for some $h \in E$. As $x[h, x]=[h, x] x$ by hypothesis,

$$
\begin{aligned}
f^{2} & =[h, x][h, x]=h^{-1} x h x[h, x]=h^{-1} x h[h, x] x \\
& =h^{-1} x h h^{-1} x h x x=1
\end{aligned}
$$

as required. This proves the lemma.

The following lemma is an easy consequence of the three-subgroup lemma.

Lemma 2.11. Suppose that $p$ is a prime, $P$ is a p-group of nilpotency class at most 2 and $\alpha \in \operatorname{Aut}(P)$ has order coprime to $p$. If $\alpha$ centralizes a maximal abelian subgroup of $P$, then $\alpha=1$.

Proof. See [17, Lemma 2.3].

For use in Lemma 2.13 and Section 6, we collect some facts in Table 1 about the action of $\mathrm{Sp}_{6}(2)$ and $\operatorname{Aut}\left(\mathrm{SU}_{4}(2)\right)$ on their irreducible eight-dimensional module $V$ over $\mathrm{GF}(2)$. Recall that $\operatorname{Aut}\left(\mathrm{SU}_{4}(2)\right) \cong \mathrm{O}_{6}^{-}(2)$ is a subgroup of $\mathrm{Sp}_{6}(2)$ [3, p. 46]. We will frequently use the fact that as an $\mathrm{SU}_{4}(2)$-module, $V$ is the natural four-dimensional $\mathrm{GF}(4) \mathrm{SU}_{4}(2)$-module regarded as a module over $\mathrm{GF}(2)$. We will often refer to this as the natural $\mathrm{SU}_{4}(2)$-module.

Proposition 2.12. Let $X \cong \operatorname{Sp}_{6}(2)$ and $Y \cong \operatorname{Aut}\left(\mathrm{SU}_{4}(2)\right)$. Assume that $V$ is the eightdimensional irreducible module for $X$ (and hence $Y$ ) over $\mathrm{GF}(2)$. Then the following properties hold. 
(i) X and $Y$ both possess exactly four conjugacy classes of involutions. In Table 1 we list the four classes of involutions and give structural information about their centralizers in both $X$ and $Y$. The involutions denoted by $u_{1}$ are the unitary transvections. The involutions denoted by $u_{3}$ and $u_{4}$ are in $\operatorname{Aut}\left(\mathrm{SU}_{4}(2)\right) \backslash \mathrm{SU}_{4}(2)$.

(ii) $X$ and $Y$ have orbits of length 135 and 120 on the nonzero elements of $V$. We call elements of the orbits nonsingular and singular vectors, respectively. Suppose that $x$ is singular and $y$ is nonsingular. Then

$$
\begin{gathered}
\left|C_{Y}(x)\right|=2^{7} \cdot 3, \quad\left|C_{X}(x)\right|=2^{9} \cdot 3 \cdot 7 . \\
C_{Y}(y) \cong 3_{+}^{1+2} \cdot \operatorname{SDih}(16), \quad C_{X}(y) \cong \mathrm{G}_{2}(2) .
\end{gathered}
$$

(iii) $X$ and $Y$ both have exactly three conjugacy classes of elements of order three. They are distinguished by their action on $V$. They have centralizers of dimension 0,2 and 4. The elements with centralizer of dimension two are 3-central and centralize only nonsingular vectors in $V^{\#}$.

(iv) For $u \in Y$ an involution, $\operatorname{dim} C_{V}(u)$ is given in column 4 of Table 1.

(v) Let $u$ be a unitary transvection. Then $C_{Y^{\prime}}(u)$ acts on $C_{V}(u) /[V, u]$ with orbits of length 1,6 and 9.

(vi) If $u$ is a unitary transvection, $F \leq C_{Y}(u)$ has order three and $C_{C_{V}(u) /[V, u]}(F) \neq 0$, then $\operatorname{dim} C_{V}(F)=2$.

(vii) For $S \in \operatorname{Syl}_{2}(Y)$ and $S_{1}=S \cap Y^{\prime}$, every $S$-invariant subspace of $V$ of dimension at least two contains $C_{V}\left(S_{1}\right)$.

(viii) $Y$ does not contain a fours group all of whose nontrivial elements are unitary transvections.

(ix) $C_{V}\left(u_{4}\right)$ is generated by nonsingular vectors.

(x) The 2-rank of $Y$ is 4 .

Proof. (i) From [3, pp. 27, 47], we see that $\operatorname{Aut}\left(\mathrm{SU}_{4}(2)\right)$ and $\mathrm{Sp}_{6}(2)$ both possess exactly four conjugacy classes of involutions.

(ii) By Witt's lemma $Y$ has exactly two orbits on the nonzero elements of $V^{\#}$ and they correspond to the singular and the nonsingular vectors. Since $2^{8}-1$ does not divide $|X|$, these orbits are also orbits under the action of $X$. Since the lengths of the orbits are 135 and 120, using [3, pp. 26, 46] we get the given structure of the stabilizers.

(iii) As $Y$ contains a Sylow 3-subgroup of $X$, we find representatives of all $X$ conjugacy classes of elements of order three in $Y$. By [3, p. 27] there are exactly three conjugacy classes of elements of order three in $Y$, which we easily distinguish by their action on $V$. We have elements which are fixed point free, which have centralizer of dimension two, and which have centralizer of dimension four. In particular, these elements are not fused in $X$.

Let $d \in Y$ have two-dimensional fixed space on $V$. Then, as $C_{V}(d)$ is perpendicular to $[V, d]$, we deduce that $C_{V}(d)$ is nonsingular (a one-dimensional nonsingular GF(4)space).

(iv) For the unitary transvection $u$ we have that $\operatorname{dim}[V, u]=2$. Suppose that $u$ is not a unitary transvection but $u \in Y^{\prime}$. Then, as $V$ supports the structure of a vector 
space over $\operatorname{GF}(4),[V, u]$ is two-dimensional and so $\operatorname{dim}[V, u]=4$. Suppose next that $u$ is an involution in $Y \backslash Y^{\prime}$ and let $P$ be the stabilizer of a maximal isotropic space $W$ of GF(4)-dimension two in $V$. Then $O_{2}(P)$ is elementary abelian of order 16 and $P / O_{2}(P) \cong \operatorname{Sym}(5) \cong \mathrm{SU}_{2}(4): 2$. Since $P$ contains a Sylow 2-subgroup of $Y$, we may suppose that $u \in P$. Furthermore, $W$ and $V / W$ are natural $\mathrm{SL}_{2}(4)$-modules. As $u \notin$ $O_{2}(P) \leq Y^{\prime}, \operatorname{dim}[W, u]=2=\operatorname{dim}[V / W, u]$. Hence $\operatorname{dim}[V, u] \geq 4$ and so as $\operatorname{dim} V=8$, we have $\operatorname{dim}[V, u]=4$.

(v) Let $u$ be a unitary transvection. Then $C_{Y^{\prime}}(u)$ acts on $C_{V}(u) /[V, u]$ as $\mathrm{GU}_{2}(2) \cong$ $\operatorname{Sym}(3) \times 3$ and has three orbits: one of length 1 , one of length 6 and one of length 9 .

(vi) From (v), a Sylow 3-subgroup $S_{1}$ of $C_{Y^{\prime}}(u)$ contains two subgroups of order three whose centralizer in $C_{V}(u) /[V, u]$ is of order four, and two which are fixed point free. As the elements of order three in $C_{Y^{\prime}}(u)$ act the same way on $[V, u]$ as on $V / C_{V}(u)$, the elements with fixed points on $C_{V}(u) /[V, u]$ have centralizer in $V$ of dimension two, as by (iii) there are no elements of order three which centralize a subspace of dimension six. Now by coprime action we get that one subgroup of order three in $S_{1}$ centralizes in $V$ a subspace of dimension four and acts fixed point freely on $C_{V}(u) /[V, u]$, one acts fixed point freely on $V$ and the other two centralize a subspace of dimension two in $V$.

(vii) Let $S \in \operatorname{Syl}_{2}(Y)$ and $S_{1}=S \cap Y^{\prime}$. Then, as $V$ is the natural four-dimensional unitary module for $Y^{\prime}, U=C_{V}\left(S_{1}\right)$ has $\mathrm{GF}(2)$-dimension two. Assume that (vii) is false and let $W$ be an $S$-invariant subspace of dimension at least two with $U \not \subset W$. Then $W>[W, S] \neq 0$ does not contain $U$ and so $[W, S]$ must have $\mathrm{GF}(2)$-dimension one by the minimal choice of $W$. Hence $[W, S] \leq C_{V}\left(S_{1}\right)=U$, which means that $W+U / U \leq C_{V / U}(S)<C_{V / U}\left(S_{1}\right)$ and this latter space has GF(4)-dimension one. It follows that $W$ has $\mathrm{GF}(2)$-dimension two. Hence $S_{0}=C_{S}(W)$ has index 2 in $S$, $S_{0} \cap S_{1}$ has order at least $2^{5}$ and this subgroup centralizes $W$ and $U$ and hence centralizes the preimage of $C_{V / U}\left(S_{1}\right)$ which has GF(4)-dimension two. However, this is an isotropic line in the unitary representation and its centralizer is elementary abelian of order $2^{4}$, a contradiction. Hence (vii) is true.

(viii) Suppose that $F=\left\langle x_{1}, x_{2}\right\rangle$ is a fours group with all nontrivial elements unitary transvections. Then, as $x_{3}=x_{1} x_{2}$ is also a unitary transvection, we get that $C_{V}\left(x_{1}\right)=$ $C_{V}\left(x_{2}\right)$. But then $C_{V}\left(x_{1}\right)$ is normalized by $\left\langle C_{Y}\left(x_{1}\right), C_{Y}\left(x_{2}\right)\right\rangle=Y$, which is impossible.

(xi) Let $y$ be a nonsingular vector. By (ii), we have that $C_{Y}(y) \cong 3_{+}^{1+2} \cdot \mathrm{GL}_{2}(3)$. This group contains an involution $u$ in $Y \backslash Y^{\prime}$. If $u$ is conjugate to $u_{3}$ (in Table 1), then $C_{Y^{\prime}}(u) \cong \operatorname{Sym}(6)$ acts transitively on $C_{V}(u)^{\#}$ and so $C_{V}(u)^{\#}$ contains only nonsingular vectors. Since $\operatorname{dim} C_{V}(u)=4$, this is impossible. Therefore $v$ is conjugate to $u_{4}$ and $y \in C_{V}(u)=[V, u]$. As $C_{C_{Y^{\prime}}(y)}(u)$ has order six, there are eight conjugates of $y$ in $C_{V}(u)$. Hence $C_{V}(u)$ is generated by nonsingular elements.

(x) From (i) we see that the centralizers of involutions $x \in Y \backslash Y^{\prime}$ have 2-rank four. Thus we only need to see that $Y^{\prime}$ has 2-rank four. This is well known and can be read from [8, Table 3.3.1].

In the next lemma the group denoted by $\left(\mathrm{SU}_{4}(2) \times 3\right): 2$ is the subgroup of index 2 in $\operatorname{Aut}\left(\mathrm{SU}_{4}(2)\right) \times \operatorname{Sym}(3)$ which is not expressible as a direct product. 
Lemma 2.13. Assume that $G$ is a group, $t \in G$ is an involution, $H=C_{G}(t)$ and $Q=$ $F^{*}(H)$ is extraspecial of order $2^{9}$. If $H / Q \cong \operatorname{Aut}\left(\mathrm{SU}_{4}(2)\right)$ or $\left(\mathrm{SU}_{4}(2) \times 3\right): 2$ and $Q /\langle t\rangle$ is the natural $F^{*}(H / Q)$-module, then $G$ has a subgroup of index 2 .

Proof. We let $S \in \operatorname{Syl}_{2}(H)$ and note that, as $Z(S)=Z(Q)=\langle t\rangle$, we have $S \in \operatorname{Syl}_{2}(G)$. Let $\bar{H}=H /\langle t\rangle$. We first show that

$$
t^{G} \cap Q=\{t\}
$$

Assume that $u \sim_{G} t$ with $u \in Q \backslash\langle t\rangle$. Then $\bar{u}$ is singular in $\bar{Q}$ and so we may suppose that $\langle\bar{u}\rangle=Z(\bar{S})$. Now $C_{Q}(u)$ contains an extraspecial group of order $2^{7}$. As a Sylow 2-subgroup of $H / Q$ is not extraspecial, we have that $t \in Q_{u}=O_{2}\left(C_{G}(u)\right.$ ). Note that $\Phi\left(Q_{u} \cap Q\right) \leq\langle u\rangle \cap\langle t\rangle=1$. Hence $Q_{u} \cap Q$ is elementary abelian. As $Q$ is extraspecial of order $2^{9}$, we deduce that $\left|Q \cap Q_{u}\right| \leq 2^{5}$. Since the 2-rank of $H / Q$ is four by Proposition 2.12(x) and $\left|C_{Q_{u}}(t)\right|=2^{8}$, we infer that $\left|Q \cap Q_{u}\right|$ is either $2^{4}$ or $2^{5}$. Furthermore, because $C_{H}(u) Q \geq S, Q \cap Q_{u}$ is a normal subgroup of $S$. We know that $\bar{Q}$ is a $\mathrm{GF}(4)$-module for $F^{*}(H / Q)$. Let $\bar{U}$ be the one-dimensional GF(4)space in $\bar{Q}$ containing $\bar{u}, U$ be its preimage in $H$ and set $R=C_{H}(U)$. Since $U$, $Q_{u} \cap Q$ and $R$ are normalized by $S$, Proposition 2.12(vii) implies that $U \leq Q_{u} \cap Q \leq R$. Assume that $\left|Q_{u} \cap Q\right|=2^{5}$. Then, as $\left(Q_{u} \cap H\right) Q / Q$ is a normal subgroup of $C_{H}(u) Q / Q$ and $C_{H}(u) Q / Q$ contains $S / Q$, we get $Z(S / Q) \leq\left(Q_{u} \cap H\right) Q / Q$. Hence there exists $w \in Q_{u} \cap H$ such that $\langle w Q\rangle=Z(S / Q)$ is the unitary transvection group centralizing $\bar{R}$. Therefore

$$
\left[Q_{u} \cap Q, w\right] \leq[R, w] \cap\left[Q_{u}, w\right] \leq\langle t\rangle \cap\langle u\rangle=1,
$$

which is impossible as $Q_{u} \cap Q$ is a maximal abelian subgroup of $Q_{u}$. Thus $\left|Q_{u} \cap Q\right|=$ $2^{4}$. Since $\left|\left(Q_{u} \cap Q\right) / U\right|=2$, we now have a contradiction to the fact that $C_{R / U}\left(C_{H}(u)\right)=$ 1 by Proposition 2.12(v). Thus (2.1) holds.

By Proposition 2.12(i), $H / Q$ has exactly two conjugacy classes of involutions not in $H^{\prime} / Q$. We choose representatives $\widetilde{x}, \widetilde{y} \in S / Q$ for these conjugacy classes and fix notation so that $C_{F^{*}(H / Q)}(\widetilde{x}) \cong \mathrm{Sp}_{4}(2)$ and $C_{F^{*}(H / Q)}(\widetilde{y}) \cong 2 \times \operatorname{Sym}(4)$. We have that $|[\bar{Q}, \widetilde{x}]|=|[\bar{Q}, \widetilde{y}]|=2^{4}$ by Proposition 2.12(iv). Let $z \in H$ with $z^{2} \in\langle t\rangle$ be such that $z Q$ is either $\widetilde{x}$ or $\widetilde{y}$. Let $T \in \operatorname{Syl}_{2}\left(C_{H}(z)\right)$. Then $T^{\prime} \cap Z(T) \leq T \cap H^{\prime}$ and $Z(T) \cap H^{\prime} \leq Q$ as $Z(T)=\left\langle z, C_{Q}(z)\right\rangle$. Thus, by (2.1), $t^{G} \cap T^{\prime} \cap Z(T)=\{t\}$. In particular, $T \in \operatorname{Syl}_{2}\left(C_{G}(z)\right)$. It follows that $z$ is not conjugate to $t$ in $G$ and that $t^{G} \cap Z(T)=\{t\}$. We record these observations as follows.

Lemma 2.14. Let $z \in S \backslash\left(S \cap H^{\prime}\right)$ be such that $z^{2} \in\langle t\rangle$ and $T \in \operatorname{Syl}_{2}\left(C_{H}(z)\right)$. Then $T \in \operatorname{Syl}_{2}\left(C_{G}(z)\right), t^{G} \cap Z(T)=\{t\}$ and $t^{G} \cap H \subset H^{\prime}$.

Now let $z_{1} \in S$ be such that $z_{1} Q=\widetilde{x}$. Since $C_{H / Q}\left(z_{1} Q\right)$ contains an element $f Q$ of order five with $f$ of order five acting fixed point freely on $\bar{Q}$, we see that $C_{Q\left\langle z_{1}\right\rangle}(f)$ has order four. Let $z \in C_{Q\left\langle z_{1}\right\rangle}(f)$ have minimal order so that $z Q=z_{1} Q$. Then $z^{2} \in\langle t\rangle$. Suppose that $g \in G$ and $z^{g} \in S \cap H^{\prime}$ is extremal in $S$. Then $C_{S}\left(z^{g}\right) \in \operatorname{Syl}_{2}\left(C_{G}\left(z^{g}\right)\right)$. Now let $T \in \operatorname{Syl}_{2}\left(C_{H}(z)\right)$. Then $T \in \operatorname{Syl}_{2}\left(C_{G}(z)\right)$ by Lemma 2.14. Hence $T^{g} \in \operatorname{Syl}_{2}\left(C_{G}\left(z^{g}\right)\right)$ 
and there is a $w \in C_{G}\left(z^{g}\right)$ such that $T^{g w}=C_{S}\left(z^{g}\right)$. Now, by Lemma 2.14, $t^{G} \cap Z\left(T^{g w}\right)=$ $\left\{t^{g w}\right\}$ and of course $t^{G} \cap Z\left(C_{S}\left(z^{g}\right)\right)=\{t\}$ as $t \in Z(H)$. Thus $g w \in H$, which is impossible as $z \in H \backslash H^{\prime}, z^{g} \in H^{\prime}$ and $z^{g w}=z^{g}$. Hence there are no extremal conjugates of $z$ in $S \cap H^{\prime}$. Since also $z^{2} \in\langle t\rangle$ and $t^{G} \cap H \subset H^{\prime}$, Lemma 2.1 implies that $G$ has a subgroup of index 2 as claimed.

\section{The finer structure of $M$}

Suppose that $G$ is a group, $Z \leq G$ has order three and set $M=N_{G}(Z)$. Assume that $C_{M}(Z)$ is similar to a 3-centralizer in a group of type $\operatorname{PSU}_{6}(2)$ or $\mathrm{F}_{4}(2)$. Let $S \in$ $\operatorname{Syl}_{3}(M)$ and $Q=F^{*}(M)=O_{3}(M)$. By definition $C_{M}(Z)$ contains a normal subgroup $R^{*}$ such that $R^{*} / Q \cong \mathrm{Q}_{8} \times \mathrm{Q}_{8}$. We let $R \in \operatorname{Syl}_{2}\left(R^{*}\right)$. Then $R^{*}=R Q$. Since, by [10, Satz III(13.7)], the commutator map from $Q / Z \times Q / Z$ to $Z$ is a $C_{M}(Z) / Z$-invariant nondegenerate symplectic form which may be negated by $M, M / Q$ embeds into $\operatorname{Out}(Q) \cong \mathrm{GSp}_{4}(3)$. Our first lemma locates $M / Q$ as a subgroup of $\mathrm{GSp}_{4}(3)$.

Lemma 3.1. The group $M / Q$ normalizes $R Q / Q$ and is isomorphic to a subgroup of the subgroup $\overline{\mathbf{M}}$ of $\mathrm{GSp}_{4}(3)$ which preserves a decomposition of the natural four-dimensional symplectic space over GF(3) into a perpendicular sum of two nondegenerate 2-spaces. Furthermore, $\mathrm{RQ} / Q$ maps to $\mathrm{O}_{2}(\overline{\mathbf{M}})$.

Proof. Consider the action of $Z(R)$ on $Q / Z$. Since $\operatorname{Out}(Q)$ is isomorphic to a subgroup of $\mathrm{GSp}_{4}(3), Z(R)$ acts as a fours group of $\mathrm{Sp}_{4}(3)$ on $Q / Z$. Let $a \in Z(R)^{\#}$. Then $Q=C_{Q}(a)[Q, a]$ and $\left[C_{Q}(a),[Q, a]\right]=1$ by the three-subgroup lemma. We may suppose that $C_{Q}(a) \neq Z$, and so $C_{Q}(a) \cong[Q, a]$ is extraspecial of order $3^{3}$. Since $R$ centralizes $a, R$ preserves this decomposition and $R_{1}=C_{R}([Q, a])$ has order eight and acts faithfully on $C_{Q}(a)$. Hence $R_{1} \cong \mathrm{Q}_{8}$ and, similarly, $R_{2}=C_{R}\left(C_{Q}(a)\right) \cong \mathrm{Q}_{8}$ with $R=R_{1} \times R_{2}$. In particular, $C_{M}(Z) / Q$ is isomorphic to a subgroup of

$$
\overline{\mathbf{M}}=\left(\mathrm{Sp}_{2}(3)<2\right) \cdot 2
$$

and $R / Q$ corresponds to the largest normal 2-subgroup of this group. It follows that $\left|O_{2}\left(C_{M}(Z)\right): R Q\right| \leq 2$. Thus $Z(R) Q / Q$ is a characteristic subgroup of $C_{M}(Z)$ and so $Z(R) Q / Q$ is normalized by $M / Q$. Finally, as $R S / Q$ is the centralizer of $Z(R) Q / Q$ in $C_{M}(Z) / Q$, we deduce that $R Q / Q$ is normalized by $M / Q$ and that $M / Q$ preserves the decomposition of $Q / Z$ as described.

For the remainder of the paper we assume that $Z$ is weakly closed in $Q$ but not in $S$ with respect to $G$. In particular, this means that $S>Q$.

By Lemma 3.1, $\bar{M}=M / Q$ is isomorphic to a subgroup of the subgroup of $\mathrm{GSp}_{4}(3)$ which preserves a decomposition of the natural four-dimensional symplectic space into a perpendicular sum of two nondegenerate 2-spaces. We now describe this subgroup of $\mathrm{GSp}_{4}$ (3). We denote it by $\overline{\mathbf{M}}$ as in Lemma 3.1. The bold face is supposed to indicate that this is a subgroup of $\mathrm{GSp}_{4}(3)$ which contains (the image of) $\bar{M}$ but may be greater than it. Similarly, $\overline{\mathbf{S}}$ is a Sylow 3 -subgroup of $\overline{\mathbf{M}}$ which contains $\bar{S}$. 
The group $\overline{\mathbf{M}}$ contains a subgroup of index 2 which is contained in $\mathrm{Sp}_{4}(3)$ and is isomorphic to the wreath product of $\mathrm{Sp}_{2}(3) \cong \mathrm{SL}_{2}(3)$ by a group of order two. For $i=1,2$, we let $\overline{\mathbf{M}_{i}} \cong \mathrm{SL}_{2}(3), \overline{\mathbf{R}_{i}}=O_{2}\left(\overline{\mathbf{M}_{i}}\right) \cong \mathrm{Q}_{8}$ and $\overline{\mathbf{S}_{i}}=\overline{\mathbf{S}} \cap \overline{\mathbf{M}_{i}}$. We let $\overline{\mathbf{t}_{1}}$ be an involution in $\overline{\mathbf{M}}$ which negates the symplectic form and normalizes $\overline{\mathbf{S}_{1}}$ and $\overline{\mathbf{S}_{2}}$. Note that, for $i=1,2, \overline{\mathbf{M}_{i}}\left\langle\overline{\mathbf{t}_{1}}\right\rangle \cong \mathrm{GSp}_{2}(3) \cong \mathrm{GL}_{2}(3)$. Next select an involution $\overline{\mathbf{t}_{2}}$ which commutes with $\overline{\mathbf{t}_{1}}$, preserves the symplectic form, normalizes $\overline{\mathbf{S}}$ and conjugates $\overline{\mathbf{M}_{1}}$ to $\overline{\mathbf{M}_{2}}$. With this notation,

$$
\overline{\mathbf{M}}=\overline{\mathbf{M}_{1} \mathbf{M}_{2}}\left\langle\overline{\mathbf{t}_{1}}, \overline{\mathbf{t}_{2}}\right\rangle .
$$

Now $\bar{S}$ has index at most 3 in $\overline{\mathbf{S}}$ as $S>Q$. Since $\overline{\mathbf{R}_{1} \mathbf{R}_{2}}=\bar{R}, M$ contains subgroups $R_{1}$ and $R_{2}$ isomorphic to $\mathrm{Q}_{8}$ such that $\left[R_{1}, R_{2}\right]=1$ and $\overline{R_{i}}=\overline{\mathbf{R}_{i}}$ for $i=1$ and 2. Moreover, $R=R_{1} R_{2}$. Let $T \in \operatorname{Syl}_{2}(M)$ with $T \geq R$. We do not yet know the index of $R$ in $T$. Thus $T$ may contain elements which map to $\overline{\mathbf{t}_{1}}, \overline{\mathbf{t}_{2}}$ or $\overline{\mathbf{t}_{1} \mathbf{t}_{2}}$. If such elements are contained in $T$ we denote these involutions by $t_{1}, t_{2}$ or $t_{1} t_{2}$ as appropriate.

LEMMa 3.2. The following properties hold:

(i) $Z=Z(S)=Z(Q), N_{G}(S) \leq M$ and $S \in \operatorname{Syl}_{3}(G)$;

(ii) $3 \leq|S / Q| \leq 3^{2}$; and

(iii) $M=N_{M}(S) R$.

Proof. Since $C_{M}(Q) \leq Q$, we have that $Z=Z(Q)=Z(S)$. Therefore $N_{G}(S) \leq N_{G}(Z)=$ $M$ and, in particular, $S \in \operatorname{Syl}_{3}\left(N_{G}(S)\right) \subseteq \operatorname{Syl}_{3}(G)$. Thus (i) holds.

Part (ii) follows directly from Lemma 3.1 and (iii) follows from the above discussion about the structure of $\bar{M}$.

For $i=1,2$, let $r_{i} \in Z\left(R_{i}\right)^{\#}$ and set $Q_{i}=\left[Q, r_{i}\right]=\left[Q, R_{i}\right]$.

LeMma 3.3. The following properties hold:

(i) $Q_{1}=\left[Q, R_{1}\right]=C_{Q}\left(R_{2}\right), Q_{2}=\left[Q, R_{2}\right]=C_{Q}\left(R_{1}\right)$ and both are normal in $S$; and

(ii) $Q_{1} \cong Q_{2} \cong 3_{+}^{1+2}$, $\left[Q_{1}, Q_{2}\right]=1$ and $Q=Q_{1} Q_{2}$.

In particular, $Q$ has exponent 3.

Proof. (i) This follows directly from the action of $M$ on $Q$ described in Lemma 3.1, as $\overline{R_{1}}$ and $\overline{R_{2}}$ are normalized by $\bar{S}$.

(ii) We have that $C_{Q}\left(r_{1}\right)$ and $\left[Q, r_{1}\right]$ commute by the three-subgroup lemma. Since, for $i=1,2,\left[Q, r_{i}\right]=\left[Q, R_{i}\right]$ has order $3^{3}$, it follows that $Q_{1} \cong 3_{+}^{1+2}$. As $r_{1} r_{2}$ inverts $Q / Z, r_{2}$ inverts $C_{Q / Z}\left(r_{1}\right)$ and so $C_{Q}\left(r_{1}\right)=Q_{2}$. In particular, $Q_{1}$ and $Q_{2}$ commute and $Q=Q_{1} Q_{2}$.

Lemma 3.4. Suppose that $X \in Z^{G} \backslash\{Z\}$ and $X \leq S$. Then, for $i=1,2,\left[X, R_{i}\right] \not \subset Q$. In particular, $\left[S / Q, R_{i}\right] \neq 1,\left|C_{Q / Z}(S)\right|=3^{2}$ and $N_{R Q}(S)=Z(R) S$.

Proof. It suffices to prove the result for $i=1$. Assume that $\left[X, R_{1}\right] \leq Q$. Since $Z$ is weakly closed in $Q$ with respect to $G$ and $X \neq Z, X \not \leq Q$. Let $Q_{X}=O_{3}\left(N_{G}(X)\right)$ and $W$ be the full preimage of $C_{Q / Z}(X)$. Since $R_{1}$ acts irreducibly on $Q_{1} / Z$ and $\left[Q_{1}, Q X\right]$ is $R_{1}$-invariant, we have $Q_{1} \leq W$ and $|W|=3^{4}$. Hence $W \cong 3 \times 3_{+}^{1+2}$. 
If $C_{W}(X)$ is nonabelian, then, as $C_{W}(X) Q_{X} / Q_{X}$ is abelian,

$$
Z=C_{W}(X)^{\prime} \leq Q_{X}
$$

Since $X$ is weakly closed in $Q_{X}$ by assumption and $Z \neq X$, this is a contradiction. Thus $[W, X]=Z, C_{W}(X)$ is abelian of order $3^{3}$ and every element of $(Z X)^{\#}$ is $G$-conjugate into $Z$. In particular, we note that $C_{W}(X)=C_{Q}(X)$ is a maximal abelian subgroup of $Q$.

Set $D=[Q, X] X$. Then, as $|Q: W|=3$ and $\left|Q: C_{Q}(X)\right|=9,[Q, X]$ has order $3^{2}$, every element of $D \backslash[Q, X]$ is $Q$-conjugate to an element of $X Z$ and, consequently, all the elements of $D \backslash[Q, X]$ are 3-central. It follows that there are exactly six elements in $D^{\#}$ which are not 3-central and together they generate $[Q, X]$. By the three-subgroup lemma, [[Q,X], $\left.C_{Q}(X)\right]=1$ and so $[Q, X] \leq C_{Q}(X)$ as $C_{Q}(X)$ is a maximal abelian subgroup of $Q$. In particular, $[Q, X] \leq C_{G}(X)$. If $[Q, X] \cap Q_{X} \neq 1$, then $\left([Q, X] \cap Q_{X}\right) X \leq D$ and has at least six nontrivial elements which are not 3central. But then $Z \leq[Q, X] \leq\left([Q, X] \cap Q_{X}\right) X$, which is a contradiction. Hence $[Q, X] \cap Q_{X}=1$ and $\left|[Q, X] Q_{X} / Q_{X}\right|=3^{2}$.

Let $w \in[Q, X]^{\#}$. Then

$$
w X \subset w Q_{X} \cap[Q, X] X
$$

and $w X \cap[Q, X]=\{w\}$. Therefore $w X \backslash\{w\}$ consists of 3-central elements. As $\left|[Q, X] Q_{X} / Q_{X}\right|=3^{2}$, there exists $w \in[Q, X]^{\#}$ such that $w Q_{X}$ is centralized by a subgroup of $C_{G}(X) / Q_{X}$ which is isomorphic to $\mathrm{Q}_{8}$. Note that there are four such elements in $[Q, X]^{\#}$. In particular, we may assume that $w$ is not 3-central. Then, for $x \in X^{\#}, w x$ is 3-central. Let $Y=\langle w x\rangle$. Then $Y Q_{X} / Q_{X}$ is centralized by a subgroup of $C_{G}(X) / Q_{X}$ isomorphic to $\mathrm{Q}_{8}$. Hence every nontrivial element of $X Y$ is 3-central. Since $w \in(X Y)^{\#}$, this is a contradiction. Therefore $\left[X, R_{1}\right] \not \leq Q$.

As, for $i=1,2,\left[X, R_{i}\right] \not \leq Q$ and $Z$ is weakly closed in $Q$ but not in $S$, we have $\left[S, R_{i}\right] \not \leq Q$. Thus $\left|C_{Q / Z}(S)\right|=3^{2}$ and $N_{R Q}(S)=Z(R) S$.

Using Lemma 3.4, we can elucidate the structure of $N_{M}(S)$ as follows.

Lemma 3.5. There are exactly five possibilities for a Sylow 2-subgroup $T$ of $M$. Moreover, one of the following holds:

(i) $T=R, N_{M}(S)=S Z(R)$ and $N_{M}(S) / S \cong 2^{2}$;

(ii) $T=R\left\langle t_{1}\right\rangle, N_{M}(S)=S Z(R)\left\langle t_{1}\right\rangle$ and $N_{M}(S) / S \cong 2^{3}$;

(iii) $T=R\left\langle t_{2}\right\rangle, N_{M}(S)=S Z(R)\left\langle t_{2}\right\rangle$ and $N_{M}(S) / S \cong \operatorname{Dih}(8)$;

(iv) $T=R\left\langle t_{1} t_{2}\right\rangle, N_{M}(S)=S Z(R)\left\langle t_{1} t_{2}\right\rangle$ and $N_{M}(S) / S \cong \operatorname{Dih}(8) ;$ and

(v) $T=R\left\langle t_{1}, t_{2}\right\rangle, N_{M}(S)=S Z(R)\left\langle t_{1}, t_{2}\right\rangle$ and $N_{M}(S) / S \cong 2 \times \operatorname{Dih}(8)$.

We let $A$ be the full preimage of $C_{Q / Z}(S)$ and note that $A=Z_{2}(S)$. As $Q_{1}$ and $Q_{2}$ are $S$-invariant, Lemma 3.4 implies that $\left(A \cap Q_{1}\right) / Z=C_{Q_{1} / Z}(S)$ and so $A$ is elementary abelian of order $3^{3}$. We now define a subgroup which will play a prominent role in future investigations. Set

$$
J=C_{S}(A)
$$


It will turn out that $J$ is the Thompson subgroup of $S$. Because, for $i=1,2, r_{i}$ inverts $Q_{i} / Z$, if $M$ contains the involution $t_{1}$, we adjust $t_{1}$ by multiplying by elements from $Z(R)$ so that $t_{1}$ inverts $A / Z$. We thus have the following lemma.

Lemma 3.6. If $t_{1} \in M$, then $t_{1}$ inverts $A$ and centralizes $Q / A$.

Lemma 3.7. The following properties hold:

(i) $|S: J|=3^{2}, J \cap Q=A$ and $S=J Q$;

(ii) $\quad N_{M}(S)=N_{M}(J)$; and

(iii) if $t_{1} \in M$, then $t_{1}$ inverts $J$ and $J$ is abelian.

Proof. We have seen that $A$ is elementary abelian of order $3^{3}$. Furthermore, by definition, $J$ is a normal subgroup of $N_{M}(S)$. Since $[S, A]=Z$, the 3-structure of $\mathrm{GL}_{3}(3)$ shows that $|S / J| \leq 3^{2}$. As $J \cap Q=C_{Q}(A)=A$, we infer that $|S: J|=3^{2}$ and $S=J Q$. Thus (i) holds.

As $A=Z_{2}(S), J=C_{S}(A)$ is normalized by $N_{M}(S)$. Also because $N_{M}(J)$ normalizes $J \cap Q=A$, we know that $N_{M}(J) \leq N_{M}(A)$. Now $N_{M}(A) \geq N_{M}(S)$ and because $N_{R}(A)=$ $Z(R)$ and $M=R N_{M}(S)$, we deduce that $N_{M}(A)=N_{M}(S)$. Therefore $N_{M}(J)=N_{M}(S)$ as claimed in (ii).

Suppose that $t_{1} \in M$. Then $t_{1}$ inverts $S / Q$, centralizes $Q / A$ and inverts $A$ by Lemma 3.6. As $t_{1} \in N_{M}(J)$ by (ii), $t_{1}$ inverts $J$ and $J$ is abelian. This proves (iii).

Note that $|J|=3^{4}$ if $|S / Q|=3$ and $|J|=3^{5}$ if $|S / J|=3^{2}$.

Lemma 3.8. $C_{G}(J) \leq J$.

Proof. As $Z \leq J$, it follows that $C_{G}(J)=C_{M}(J)$. Now $C_{G}(J)$ centralizes $A=J \cap Q$ and it follows from Lemma 2.11 that $C_{M}(J)=C_{S}(J) \leq J$.

Lemma 3.9. Every element of $Q$ is conjugate in $M$ to an element of $A$.

Proof. It suffices to prove that every element of $Q / Z$ is conjugate to an element of $A / Z$. Let $w \in Q / Z$. Then $w=x_{1} x_{2}$ where $x_{i} \in Q_{i} / Z$ by Lemma 3.3(ii). Since, for $i=1,2,\left(A \cap Q_{i}\right) / Z$ has order three and $R_{i}$ acts transitively on $Q_{i} / Z$, there exists $u_{i} \in R_{i}$ such that $w^{u_{1} u_{2}}=x_{1}^{u_{1}} x_{2}^{u_{2}} \in A / Z$. This proves the claim.

\section{The structure of the normalizer in $G$ of $J$}

For the remainder of the paper assume the hypothesis of Theorem 1.2. Thus $M, Q, S$ and $Z$ are as in Section 3 and additionally $Z$ is weakly closed in $Q$ and not in $M$ (with respect to $G)$. In this section we determine the possible structures of $N_{G}(J)$.

LemMa 4.1. If $Z$ is not weakly closed in $J$, then $J$ is elementary abelian and coincides with the Thompson subgroup of $S$. In particular, $N_{G}(J)$ controls fusion in $J$. 
Proof. Choose $X \in Z^{G}$ with $X \neq Z$ and $X \leq J$. Set $K=A X$. As $Z$ is weakly closed in $Q$ and $J=C_{S}(A), K$ is elementary abelian of order $3^{4}$. In particular, if $|J|=3^{4}$, then $K=J$ is elementary abelian.

Suppose that $|J|=3^{5}$. Then $|J: K|=3$ and $|S / Q|=3^{2}$. We claim that $J$ is abelian. Set $Q_{X}=O_{3}\left(N_{G}(X)\right)$. As $K$ has index 3 in $J, K$ is normal in $J$ and, as $[Q, X] \leq A, K$ is normalized by $Q$. Therefore $K$ is normal in $S=J Q$ by Lemma 3.7(i). If $C_{S}(X)=K$, then $\left|X^{S}\right|=3^{3}$ and, in particular, every element of $K$ which is not conjugate to an element of $Z$ is contained in $A$. Now $K \cap Q_{X}$ has order either $3^{2}$ or $3^{3}$ and, so, as $X$ is weakly closed in $Q_{X}, K \cap Q_{X}$ is generated by elements which are not conjugate to elements of $Z$. It follows that $X \leq K \cap Q_{X} \leq A$, and this contradicts $X \not \leq Q$. Therefore $C_{S}(X) \neq K$. If $C_{S}(X) \not J J$, then $Z=\left[A, C_{S}(X)\right] \leq C_{S}(X)^{\prime} \leq Q_{X}$, and this contradicts the fact that $X$ is weakly closed in $Q_{X}$. So $C_{S}(X) \leq J$. But then $K \leq Z(J)$ and so $J$ is abelian as claimed.

Suppose that $B \leq S$ is abelian and $|B| \geq|J|$. As $|B \cap Q| \leq 3^{3}$, we have $B Q=S$ and so $(B \cap Q) / Z \leq C_{Q / Z}(S)=A / Z$. Thus $B \leq C_{S}(A)=J$. Hence $J$ is the Thompson subgroup of $S$. As $J$ is abelian and weakly closed in $S$, it follows from [1, (37.6)] that $N_{G}(J)$ controls fusion in $J$. In particular, $X$ and $Z$ are conjugate in $N_{G}(J)$. Since $\Phi(J) \leq A$, $X \not \leq \Phi(J)$ and hence $Z \not \leq \Phi(J)$. Therefore $Z(S) \cap \Phi(J)=1$. As $\Phi(J)$ is normal in $S$, we get $\Phi(J)=1$ and $J$ is elementary abelian. This completes the proof of the lemma.

Lemma 4.2. Assume that $Z$ is not weakly closed in $J$ and set $J_{0}=\left\langle Z^{N_{G}(J)}\right\rangle$. Then:

(i) $\left|Z^{N_{G}(J)}\right|=10$ and, if $X \in Z^{N_{G}(J)}$ with $X \neq Z,\left|X^{Q}\right|=3^{2}$;

(ii) $N_{G}(J)$ acts 2-transitively on $Z^{N_{G}(J)}$; and

(iii) $\left|J_{0} Q / Q\right|=3$ and $J_{0} Q / Q$ is normalized by $N_{M}(S) / Q$.

Proof. Let $\mathcal{Y}=Z^{N_{G}(J)}$ and $X \in \mathcal{Y}$ with $X \neq Z$. Of course $X \not \leq Q$ as $Z$ is weakly closed in $Q$. By Lemma 3.4, $C_{Q}(X)=A$ has order $3^{3}$ and, in particular (as $J$ is abelian), $X^{S}=X^{J Q}=X^{Q}$ has order $3^{2}$ and so $|\mathcal{Y}| \equiv 1(\bmod 9)$. Observe that

$$
|\mathcal{Y}|=\left|N_{G}(J)\right| /\left|N_{M}(J)\right|=\left|N_{G}(J) / J\right| /\left|N_{M}(J) / J\right| .
$$

As $|J|=3^{4}$ or $3^{5}$ and $J$ is self-centralizing and elementary abelian by Lemmas 3.8 and $4.1,\left|N_{G}(J) / J\right|$ divides $\left|\mathrm{GL}_{5}(3)\right|$. If $|J|=3^{4}$, then, as no subgroup of order three in $A$ which is not $Z$ is conjugate to $Z, J$ contains at most 28 conjugates of $Z$. This means that $|\mathcal{Y}|=10,19$ or 28 . On the other hand, $\left|\mathrm{GL}_{4}(3)\right|_{3^{\prime}}=2^{9} \cdot 5 \cdot 13$ and so in this case $|\mathcal{Y}|=10$. So assume from now on that $|J|=3^{5}$. Then $J$ contains 121 subgroups of order three, and 12 of these are contained in $A$ and are not conjugate to $Z$ as $Z$ is weakly closed in $Q$. Since $\left|\mathrm{GL}_{5}(3)\right|_{3^{\prime}}=2^{10} \cdot 5 \cdot 11^{2} \cdot 13$ and $|\mathcal{Y}| \equiv 1 \bmod 9$, the only candidates for $|\mathcal{Y}|$ are 10, 55 and 64. We recall from Lemma 3.7 that $\left|N_{M}(J) / J\right|=2^{i} \cdot 3^{2}$ where $i \in\{2,3,4\}$ and, if $t_{1} \in M$, then $t_{1} J \in Z\left(N_{G}(J) / J\right)$ and $t_{1}$ inverts every element of $J$ by Lemma 3.7(iii). In particular, $t_{1}$ normalizes every member of $\mathcal{Y}$.

Suppose that $|\boldsymbol{y}|=55$. Then, by Lemma 3.7(ii),

$$
\left|N_{G}(J) / J\right|=\left|N_{G}(J): N_{M}(J)\right|\left|N_{M}(J): J\right|=2^{i} \cdot 3^{2} \cdot 5 \cdot 11
$$


where $i \in\{2,3,4\}$. Let $E \in \operatorname{Syl}_{11}\left(N_{G}(J) / J\right)$. As the normalizer of a cyclic subgroup of order 11 in $\mathrm{GL}_{5}(3)$ has order $2 \cdot 5 \cdot 11^{2}$, the normalizer in $N_{G}(J) / J$ of $E$ has order dividing 110. In particular, $E$ is not normal in $N_{G}(J) / J$. If $\left|N_{M}(J)\right|_{2}=2^{4}$, then $t_{1} J$ normalizes $E$. So in every case the number of conjugates of $E$ in $N_{G}(J) / J$ divides $2^{3} \cdot 3^{2} \cdot 5$ and is divisible by $2^{2} \cdot 3^{2}$ and this is impossible as it must also be congruent to $1 \bmod 11$.

Suppose that $|\mathcal{Y}|=64$. Then $\left|N_{G}(J) / J\right|=2^{j} \cdot 3^{2}$ where $j \in\{8,9,10\}$. In particular, $N_{G}(J)$ is soluble. Since $|\mathcal{Y}|=64$, we have that $J=\langle\mathcal{Y}\rangle$. If $1 \neq K \leq J$ is normal in $N_{G}(J)$, then $K$ is normal in $S$ and consequently $Z \leq K$. But then $\mathcal{Y} \subseteq K$ and so $K=J$. Thus $N_{G}(J)$ acts irreducibly on $J$. Since $|J|=3^{5}$ and $N_{G}(J) / J$ is not abelian, Schur's lemma implies that $\left|Z\left(N_{G}(J) / J\right)\right|$ divides 2 and, additionally, $O_{3}\left(N_{G}(J) / J\right)=1$. Let $L=O_{3,2}\left(N_{G}(J)\right)$. By Clifford's theorem [6, Theorem 4.3.1], $J$ is completely reducible as an $L$-module and $N_{G}(J)$ acts transitively on the homogeneous summands of $J$ restricted to $L$. Since $J$ has dimension five as a $\operatorname{GF}(3) N_{G}(J)$-module, and 5 does not divide $\left|N_{G}(J)\right|, J$ is homogeneous as an $L$-module. It follows that $J$ is either a direct sum of five one-dimensional $L$-modules or is irreducible as an $L$-module. It the first case, $\left[L, N_{G}(J)\right] \leq J, O_{3}\left(N_{L}(J) / J\right) \neq 1$ and this contradicts $O_{3}\left(N_{G}(J)\right)=J$. Thus $J$ is an irreducible $L$-module. However, the degrees of irreducible $L / Q$-modules over the algebraic closure of GF(3) are all powers of 2 [11, Theorem 15.13] and this again implies that $L / C_{L}(J)$ is cyclic, and $O_{3}\left(N_{G}(J)\right)>J$, again a contradiction. Thus $|\mathcal{Y}| \neq 64$.

Since $|\mathcal{Y}| \neq 55$ or 64 , we must have $|\mathcal{Y}|=10$ as claimed in the first part of (i). Because $C_{Q}(X)=A$, the remaining parts of (i) also hold.

Part (ii) follows directly from (i).

Now with $J_{0}=\left\langle Z^{N_{G}(J)}\right\rangle$, we have that $J_{0} Q=\left\langle X^{Q}\right\rangle Q=X Q$ is normalized by $N_{M}(S)$ and $\left|J_{0} Q / Q\right|=|X Q / Q|=3$. This is (iii).

Lemma 4.3. Assume that $X \in Z^{G}$ with $X \leq S$. Then $X \leq J$. In particular, $Z$ is not weakly closed in $J$.

Proof. Suppose that $X \leq S$ and $X \not \leq J$. Then $[A, X]=Z$ and $\left|C_{A}(X)\right|=3^{2}$. By Lemma 3.4, $X Q / Q$ acts nontrivially on both $R_{1} Q / Q$ and $R_{2} Q / Q$ and so $C_{A}(X)=$ $C_{Q}(X)$. On the other hand, $A X$ is normalized by $Q$ and so $A X$ contains at least, and hence exactly, 28 conjugates of $Z$. In particular, $C_{A}(X) X$ contains 10 conjugates of $Z$ and three subgroups of order three which are not conjugate to $Z$. Set $Q_{X}=$ $O_{3}\left(N_{G}(X)\right)$. Then the only conjugate of $Z$ contained in $C_{A}(X) X \cap Q_{X}$ is $X$. Since the subgroups of order three in $C_{A}(X)$ which are not conjugate to $Z$ generate $C_{A}(X)$, we get $C_{A}(X) X \cap Q_{X}=X$. So $\left|C_{A}(X) Q_{X} / Q_{X}\right|=3^{2}$. By Lemma 3.4, two of the nontrivial cyclic subgroups of $C_{A}(X) Q_{X} / Q_{X}$ are not images of elements from $Z^{G}$. Since $C_{A}(X) X$ contains only three subgroups of order three which are not conjugate to $Z$, we have a contradiction. Therefore, if $X \in Z^{G}$ and $X \leq S$, then $X \leq J$ as claimed.

Set

$$
J_{0}=\left\langle Z^{N_{G}(J)}\right\rangle
$$


By Lemmas 3.4, 4.2 and 4.3, $\left|J_{0} Q / Q\right|=3, J_{0} \cap Q=A$ and $J_{0} Q / Q$ does not centralize either $R_{1} Q / Q$ or $R_{2} Q / Q$. In particular, $\left|J_{0}\right|=3^{4}$. We record these facts in the first part of the next lemma.

LEMma 4.4. The following properties hold:

(i) $\left|J_{0}\right|=3^{4},\left|J_{0} Q / Q\right|=3, J_{0} \cap Q=A$ and $J_{0} Q / Q$ acts nontrivially on both $R_{1} Q / Q$ and $R_{2} Q / Q$

(ii) $N_{G}(J)=N_{G}\left(J_{0}\right)$; and

(iii) $C_{G}\left(J_{0}\right)=C_{G}(J)=J$.

Proof. From the construction of $J_{0}$ we have $N_{G}\left(J_{0}\right) \geq N_{G}(J)$. Since $N_{G}(J)$ is transitive on the subgroups of $J$ which are $G$-conjugate to $Z$, we get that $N_{G}\left(J_{0}\right)=N_{G}(J) N_{M}\left(J_{0}\right)$. Hence, as $N_{M}\left(J_{0} Q\right)=N_{M}(S) \leq N_{G}(J)$, (ii) holds. Obviously $C_{G}\left(J_{0}\right) \leq C_{M}\left(J_{0}\right) \leq$ $C_{M}(A)=J$ so (iii) also holds.

Define

$$
F=O^{2}\left(N_{G}(J)\right)\left\langle r_{2}\right\rangle
$$

Note that $F$ is a group as $r_{2}$ normalizes $S$ and hence $J$.

THEOREM 4.5. The following properties hold:

(i) The action of $N_{G}(J)$ on $J_{0}$ preserves a nondegenerate quadratic form q of--type.

(ii) $Z^{N_{G}(J)}$ is the set of singular one-dimensional subspaces with respect to q.

(iii) $N_{G}(J) / J \cong 2 \times \operatorname{Sym}(6)$ or $\operatorname{Sym}(6)$.

(iv) $F / J \cong \operatorname{Sym}(6)$ and $\left|\left[J, r_{2}\right]\right|=3$. Furthermore, $\left[r_{2}, J\right] \leq J_{0}$ and $[J, F] \leq J_{0}$.

Proof. Let $X \in Z^{G} \backslash\{Z\}$ with $X \leq J$. For $i=1,2$, using Lemma 4.4(i), we have that $\left[J_{0}, Q_{i} / Z\right] \neq 1$ and so, as $\left[J_{0}, Q_{i}\right]$ is normal in $Q_{i}$, we get $\left|\left[J_{0}, Q_{i}\right]\right|=3^{2}$ and $\left[J_{0}, Q_{i}, Q_{i}\right]=Z$. Furthermore, $\left[J_{0}, Q_{i}\right]$ is centralized by $Q_{3-i}$. Hence $\left[J_{0}, Q_{i}\right]=$ $C_{J_{0}}\left(Q_{3-i}\right)$. By Lemma 2.4, there exists a nondegenerate quadratic form q on $J_{0}$ which is preserved by $Q$ and such that the elements of $X$ are singular vectors. It follows that with respect to q, the elements of $\cup X^{Q}$ are singular. Furthermore, as $Z=C_{J_{0}}(Q), Z$ also consists of singular vectors. Now with respect to the bilinear form $\mathrm{f}$ associated with q, none of the nontrivial elements of $\cup X^{Q}$ is perpendicular to a nontrivial element of $Z$. It follows that $X Z$ contains exactly two singular subspaces, namely $X$ and $Z$. Since $N_{G}(J)$ acts 2-transitively on $Z^{N_{G}(J)}$ by Lemma 4.2(ii), if $X, Y \in Z^{N_{G}(J)}$ with $X \neq Y$, then $X Y$ contains exactly two members of $Z^{N_{G}(J)}$. Now suppose that $a \in Q \backslash J$ is such that $a J$ acts quadratically on $J_{0}$. For $X \in Z^{N_{G}(J)} \backslash Z$ we know that $[X, a] \neq 1$ and hence $|[X, a]|=3$ as $a$ acts quadratically on $J_{0}$. It follows that $X[X, a]=X X^{a}$ contains three members of $Z^{N_{G}(J)}$, namely, $X, X^{a}$ and $X^{a^{2}}$. This contradiction shows that no nontrivial element of $S / J$ acts quadratically on $J_{0}$. If q were of +-type, this would not be the case. Hence q is of --type. We now have that $Z^{N_{G}(J)}$ is the set of singular 1-spaces in $J_{0}$ with respect to q. Since $N_{G}(J)$ preserves this set, $N_{G}(J) / J$ is isomorphic to a subgroup of $\mathrm{CO}_{4}^{-}(3)$, the group preserving q up to negation, by Lemma 2.3. Since $N_{N_{G}(J)}(Z)$ has index 10 in $N_{G}(J)$, we deduce that $\left|N_{G}(J)\right|=2^{i}$. $5 \cdot 3^{2}$ where $i \in\{2,3,4\}$ by Lemma 3.5. In particular, $O^{2}\left(N_{G}(J) / J\right) \cong \Omega_{4}^{-}(3) \cong \operatorname{Alt}(6)$. 
Now we see that $N_{O^{2}\left(N_{G}(J)\right)}(S) / S$ is a cyclic group of order four. Consequently, of the five possibilities for the structure of $N_{M}(S) / S$ given in Lemma 3.5, only possibilities (iii)-(v) survive and $N_{M}(S) / N_{O^{2}\left(N_{G}(J)\right)}(S)$ is elementary abelian.

Now let $C=\mathrm{CO}_{4}^{-}(3)$. If $D \in \operatorname{Syl}_{3}(X)$ and $Y \in \operatorname{Syl}_{2}\left(N_{C}(D)\right)$, then $Y \cong 2 \times \operatorname{SDih}(16)$ and so, using the structure of $N_{M}(S) / S$ given in Lemma 3.5(iii)-(v), we infer that $N_{G}(J) / J \cong \operatorname{Sym}(6)$ or $\mathrm{GO}_{4}^{-}(3) \cong 2 \times \operatorname{Sym}(6)$. We have now established (i)-(iii).

We know that $S / Q=J Q / Q$ is centralized by $r_{2}$ and that $\left[Q, r_{2}\right]=Q_{2}$. It follows that $\left[J, r_{2}\right] \leq Q_{2} \cap J=A \cap Q_{2}$ and, as $\left[A \cap Q_{2}, r_{2}\right]$ has order three, $\left[J, r_{2}\right]=\left[A, r_{2}\right]$ is a noncentral cyclic subgroup of $Q$. In particular, $\left[J, r_{2}\right] \leq A \leq J_{0}$. Since $\left|\left[J_{0}, r_{2}\right]\right|=3$ we get that $r_{2}$ has determinant -1 on $J_{0}$. Hence $r_{2} \notin O^{2}\left(N_{G}(J)\right)$ and so we conclude that $F / J \cong \operatorname{Sym}(6)$ and that all the parts of (iv) hold.

As a corollary to Theorem 4.5 we record the following observation.

Corollary 4.6. There are exactly three possibilities for a Sylow 2-subgroup $T$ of $M$ :

(i) $T=R\left\langle t_{2}\right\rangle, N_{M}(S)=S Z(R)\left\langle t_{2}\right\rangle$ and $N_{M}(S) / S \cong \operatorname{Dih}(8)$;

(ii) $T=R\left\langle t_{1} t_{2}\right\rangle, N_{M}(S)=S Z(R)\left\langle t_{1} t_{2}\right\rangle$ and $N_{M}(S) / S \cong \operatorname{Dih}(8)$; and

(iii) $T=R\left\langle t_{1}, t_{2}\right\rangle, N_{M}(S)=S Z(R)\left\langle t_{1}, t_{2}\right\rangle$ and $N_{M}(S) / S \cong 2 \times \operatorname{Dih}(8)$.

In particular, $Q / Z$ is a chieffactor in $M$.

Proof. The first three statements are readily deduced from the structure of $N_{G}(J) / J$ and so we only need to explain the fact that $Q / Z$ is a chief factor. For this we simply note that $t_{2} \in M$ or $t_{1} t_{2} \in M$ in all cases.

Theorem 4.7. If $N_{M}(S) / S \cong \operatorname{Dih}(8)$, then $G \cong \operatorname{PSU}_{6}(2)$ or $\operatorname{PSU}_{6}(2): 3$.

Proof. Since $N_{M}(S) / S \cong \operatorname{Dih}(8), N_{G}(J) / J \cong \operatorname{Sym}(6)$ by Theorem 4.5(iii). From Theorem 4.5(iv), $\left[J, r_{2}\right] \leq J_{0}$ and so we infer that $J / J_{0}$ is centralized by $N_{M}(J)$. If $J>J_{0}$, then, by Lemma 2.2, $G$ has a normal subgroup $G^{*}$ of index 3 . If $J=J_{0}$, then set $G=G^{*}$. Now $M \cap G^{*}$ satisfies the hypothesis of Theorem 2.9. Hence $G^{*} \cong \operatorname{PSU}_{6}(2)$ and this proves the theorem.

In light of Theorem 4.7 and Corollary 4.6, we may henceforth assume that $N_{M}(S)=$ $S Z(R)\left\langle t_{1}, t_{2}\right\rangle$. In particular, from Theorem 4.5,

$$
\begin{gathered}
N_{M}(S) / S \cong 2 \times \operatorname{Dih}(8) ; \\
N_{G}(J) / J \cong 2 \times \operatorname{Sym}(6) ; \text { and } \\
C_{F / J}\left(r_{2} J\right) \cong 2 \times \operatorname{Sym}(4) .
\end{gathered}
$$

Furthermore, as $t_{1}$ inverts $J$, we have $t_{1} J \in Z\left(N_{G}(J) / J\right)$.

LeMma 4.8. We have

$$
\begin{gathered}
C_{S}\left(Q_{1}\right)=C_{S}\left(R_{1}\right)=C_{S}\left(Q_{1} R_{1}\right), \\
C_{J}\left(Q_{1}\right)=C_{J}\left(R_{1}\right)=C_{J}\left(Q_{1} R_{1}\right)
\end{gathered}
$$

and $\left|J: C_{J}\left(Q_{1}\right)\right|=3^{2}$. 
Proof. We have that $\left[Q_{1}, C_{S}\left(R_{1}\right)\right]$ is $R_{1}$-invariant and is a proper subgroup of $Q_{1}$. Therefore $\left[Q_{1}, C_{S}\left(R_{1}\right)\right] \leq Z$. Hence $\left[Q_{1}, C_{S}\left(R_{1}\right), R_{1}\right]=1$ and $\left[C_{S}\left(R_{1}\right), R_{1}, Q_{1}\right]=1$ and thus the three-subgroup lemma implies that $\left[Q_{1}, R_{1}, C_{S}\left(R_{1}\right)\right]=1$. Since $Q_{1}=\left[Q_{1}, R_{1}\right]$, we have $C_{S}\left(R_{1}\right) \leq C_{S}\left(Q_{1}\right)$. Now, as $Q_{1}$ is normal in $S$ and extraspecial of order $3^{3},\left|S: C_{S}\left(Q_{1}\right) Q_{1}\right|=3$, and so $\left|C_{S}\left(Q_{1}\right)\right|=3^{4}$ if $|S|=3^{7}$ and $\left|C_{S}\left(Q_{1}\right)\right|=3^{3}$ if $|S|=3^{6}$. Since $R_{1}$ centralizes $Q_{2}$, we have $C_{S}\left(R_{1}\right)=C_{S}\left(Q_{1}\right)=Q_{2}$ if $|S|=3^{6}$. If $|S|=3^{7}$, then, as $R_{1} Q$ is normalized by $R_{1} S$, we have $\left|S / C_{S}\left(R_{1}\right) Q\right|=3$ and hence the equality $C_{S}\left(Q_{1}\right)=C_{S}\left(R_{1}\right)$ holds in this case as well. Now $C_{J}\left(Q_{1}\right)=C_{J}\left(R_{1}\right)=C_{J}\left(Q_{1} R_{1}\right)$.

As $J$ normalizes $R_{1} Q$ and does not centralize $R_{1} Q / Q$ by Lemma 3.4, $Q_{1}$ is normalized by $J$. Since $J$ is abelian and $J \cap Q_{1}=A \cap Q_{1}$, we now have that $\mid J$ : $C_{J}\left(Q_{1}\right) \mid=3^{2}$.

Notice that $r_{1} J$ and $r_{2} J$ are conjugate in $N_{G}(J) / J$ (by $t_{2} J$, for example) and

$$
\left\langle r_{1}, r_{2}, Q_{1}\right\rangle J / J \cong 2 \times \operatorname{Sym}(3) .
$$

In particular, $r_{1} \in F$.

Let $U \leq F$ be chosen so that $\left\langle r_{1}, r_{2}, Q_{1}\right\rangle J \leq U$ and $U / J \cong \operatorname{Sym}(5)$.

Lemma 4.9. If $J \neq J_{0}$, then $\left|C_{J}(U)\right|=3$ and $\left|C_{J}(U)^{F}\right|=\left|C_{J}(U)^{N_{G}(J)}\right|=6$.

Proof. Since $O^{2}(U)$ is generated by two conjugates of $Q_{1} J$, and $\left|J: C_{J}\left(Q_{1}\right)\right|=3^{2}$ by Lemma 4.8, $\left|C_{J}\left(O^{2}(U)\right)\right| \geq 3$. As the elements of order five in $U$ act fixed point freely on $J_{0}$, we have $C_{J}\left(O^{2}(U)\right) \cap J_{0}=1$. Thus $\left|C_{J}\left(O^{2}(U)\right)\right|=3$ and, as $r_{2}$ centralizes $J / J_{0}$ and normalizes $C_{J}\left(O^{2}(U)\right)$, we get that $C_{J}\left(O^{2}(U)\right)=C_{J}(U)$. Since $|F: U|=6, U$ is a maximal subgroup of $F$ and $t_{1}$ inverts $J$, we learn that $\left|C_{J}(U)^{F}\right|=\left|C_{J}(U)^{N_{G}(J)}\right|=6$.

Lemma 4.10. Suppose that $B \leq J_{0}$ with $|B|=3^{3}$. Then $B$ contains a conjugate of $Z$.

Proof. Recall that $J_{0}$ is a nondegenerate quadratic space by Theorem 4.5(i). Hence this result follows because every subgroup of order $3^{3}$ in $J_{0}$ contains a singular vector and the singular 1-spaces in $J_{0}$ are $G$-conjugate to $Z$.

We now fix some further notation. Let $W=C_{F}\left(r_{2}\right)$. By the Frattini argument, $W J / J \cong C_{F / J}\left(r_{2} J\right)$ and so $W J / J \cong 2 \times \operatorname{Sym}(4)$ and $J \cap W$ has index 3 in $J$ by Theorem 4.5(iv).

If $J=J_{0}$, set $\tau=1$, whereas if $J>J_{0}$, select $\tau \in C_{J}(U)^{\#}$.

Suppose that $J>J_{0}$. Then $\tau \neq 1$. Let

$$
\mathcal{T}=\tau^{F}=\left\{\tau_{1}=\tau, \ldots, \tau_{6}\right\}
$$

be the six $F$-conjugates of $\tau$. As $\left[J, r_{2}\right]$ has order three by Theorem 4.5(iv), $r_{2}$ acts as a transposition on $\mathcal{T}$ and $r_{2}$ centralizes $\tau$ (as $r_{2} \in U$ ). Since $W J / J \cong 2 \times \operatorname{Sym}(4)$ and $W$ has orbits of length 2 and 4 on $\mathcal{T}$, after adjusting notation if necessary, we may assume that $\tau^{W}=\left\{\tau_{1}, \tau_{2}, \tau_{3}, \tau_{4}\right\}$ and $\tau_{5}^{r_{2}}=\tau_{6}$. We further fix notation so that $Q_{1}$ acts as $\left\langle\left(\tau_{2}, \tau_{3}, \tau_{4}\right)\right\rangle$ and, since $r_{1}$ is conjugate to $r_{2}$ in $N_{G}(J)$ and inverts $Q_{1} J / J$, we may suppose that $r_{1}$ induces the transposition $\left(\tau_{2}, \tau_{3}\right)$ on $\tau^{W}$. 
For $1 \leq i \leq 4$, define

$$
J_{i}=\left\langle\tau_{j} \mid 1 \leq j \leq 4, i \neq j\right\rangle .
$$

Then each $J_{i}$ is centralized by $r_{2}$ and is a hyperplane of $C_{J}\left(r_{2}\right)$. Further,

$$
J_{i} \cap J_{j}=\left\langle\tau_{k} \mid 1 \leq k \leq 4, k \notin\{i, j\}\right\rangle .
$$

Let $\rho \in\left[J, r_{2}\right]^{\#}$. Then $\rho \in\left(A \cap Q_{2}\right) \backslash Z$ as $\left|\left[J, r_{2}\right]\right|=3$. Since $\left[J, r_{1}\right] \leq A \cap Q_{1}$, we know that $\left[\rho, r_{1}\right]=1$. From the choice of $\tau$ and $\rho$, we have that $\left\langle Q_{1}, r_{1}\right\rangle$ and $\langle\tau, \rho\rangle$ commute.

We now select and fix once and for all

$$
\rho \in\left[A \cap Q_{2}, r_{2}\right]^{\#} .
$$

For $J_{0}=J$ we must define the groups $J_{1}, J_{2}, J_{3}$ and $J_{4}$ differently. Set $J_{1}=C_{A}\left(r_{2}\right)=$ $A \cap Q_{1}$. So $J_{1}$ is normalized by $\left\langle r_{1}, r_{2}, Q_{1}, J\right\rangle$ which has index 4 in $W$. Observing that $Z$ is centralized by the Sylow 3-subgroup $S$ of $F$ and $\langle W, S\rangle=F$ yields that $W$ is not contained in $M$. As $Z$ is the unique element of $Z^{G}$ contained in $J_{1}$, we have $J_{1}^{W}=\left\{J_{1}, J_{2}, J_{3}, J_{4}\right\}$ and $W$ acts 2-transitively on $J_{1}^{W}$. As $r_{1}$ and $r_{2}$ are $M$-conjugate, all the elements in $J_{1} \backslash Z$ are conjugate to $\rho$. Therefore, as all the subgroups $J_{i}$ are centralized by $r_{2},\left|J_{i} \cap J_{j}\right|=3$ for $1 \leq i<j \leq 4$ and these intersections are conjugate to $\langle\rho\rangle$. We capture some of the salient properties of these subgroups in the next lemma.

LeMma 4.11. For $1 \leq i \leq 4, J_{i} \leq C_{G}\left(r_{2}\right)$ and $N_{N_{G}(J)}\left(J_{i}\right)$ contains a Sylow 3-subgroup of $N_{G}(J)$.

Proof. If $J>J_{0}$, this is transparent from the construction of $J_{i}$. If $J=J_{0}$, we have already mentioned that the subgroups commute with $r_{2}$. Also $J_{1}=A \cap Q_{1}$ is normalized by $S$ and as $J_{i}, 2 \leq i \leq 4$, are $W$-conjugates to $J_{1}$, we have $N_{N_{G}(J)}\left(J_{i}\right)$ contains a Sylow 3-subgroup of $N_{G}(J)$.

Note also that when $|J|=3^{5}, \rho \in\left\langle\left[\tau_{5}, r_{2}\right]\right\rangle$. It follows that $\left\langle\tau_{5}, \tau_{6}\right\rangle$ contains $\rho$ in this case. When $J=J_{0}$, of course we have $\tau_{1}=1$. Thus to handle the two possible cases simultaneously we consider the group $\left\langle\tau_{5}, \rho\right\rangle$.

Lemma 4.12. $\left\langle\tau_{5}, \rho\right\rangle$ is centralized by $J Q_{1} R_{1}$. Furthermore, $C_{G}\left(\left\langle\tau_{5}, \rho\right\rangle\right) \not M$.

Proof. Set $X=\left\langle\tau_{5}, \rho\right\rangle$. If $|J|=3^{4}$, then $X=\langle\rho\rangle \leq A \cap Q_{2}$ and the lemma holds. So suppose that $|J|=3^{5}$. Then $X=\left\langle\tau_{5}, \tau_{6}\right\rangle$ is centralized by $J$. Further, as $\left\{\tau_{5}, \tau_{6}\right\}$ is a $W$ orbit and $Q_{1} \leq C_{F}\left(r_{2}\right) \leq W, Q_{1}$ centralizes $X$. Since $C_{J}\left(Q_{1}\right)=C_{J}\left(R_{1}\right)$ by Lemma 4.8, we now have $\left[X, R_{1}\right]=1$ and this completes the proof.

Notice that $\left\langle\tau_{5}, \rho\right\rangle$ is centralized by a subgroup of index 2 in $W$ and so $C_{G}\left(\left\langle\tau_{5}, \rho\right\rangle\right)$ is not contained in $M$.

LEMMa 4.13. The following properties hold:

(i) $C_{M}(\rho)=J Q_{1} R_{1}\left\langle r_{2} t_{1}\right\rangle$; and

(ii) if $J>J_{0}, C_{M}\left(\left\langle\tau_{5}, \rho\right\rangle\right)=J Q_{1} R_{1}$. 
Proof. We calculate that $C_{M}(\rho)$ contains $J Q_{1} R_{1}\left\langle r_{2} t_{1}\right\rangle$. Because $J Q_{1} R_{1}\left\langle r_{2} t_{1}\right\rangle$ covers $C_{M / Q}(\rho Z)$, (i) holds.

By Lemma $4.12,\left\langle\tau_{5}, \rho\right\rangle$ is centralized by $J Q_{1} R_{1}$. Since, by Lemma 3.7(iii), $r_{2} t_{1}$ conjugates $\left\langle\tau_{5}\right\rangle$ to $\left\langle\tau_{6}\right\rangle$, part (ii) follows from (i).

Lemma 4.14. $Z$ is the unique $G$-conjugate of $Z$ in $\left\langle\tau_{5}, \rho, Z\right\rangle$.

Proof. Since $Z$ is weakly closed in $Q, Z$ is the unique conjugate of $Z$ in $\langle Z, \rho\rangle$. Also, as $\tau_{5}$ is not contained in $J_{0}$ and all the $G$-conjugates of $Z$ in $J$ are contained in $J_{0}$, there are no $G$-conjugates of $Z$ in $\left\langle\tau_{5}, \rho, Z\right\rangle \backslash\langle\rho, Z\rangle$. This proves the claim.

Lemma 4.15. Assume that $J>J_{0}$. Then $N_{G}\left(\left\langle r_{1}, r_{2}\right\rangle\right) / C_{G}\left(\left\langle r_{1}, r_{2}\right\rangle\right) \nRightarrow \operatorname{Sym}(3)$.

Proof. Let $U=\left\langle r_{1}, r_{2}\right\rangle$. By the Frattini argument, $N_{M}(U)$ covers $M / Q$ and $C_{M}(U)$ contains all the elements of order three in $N_{M}(U)$. Hence, as $J>J_{0}$, we have $\left|C_{M}(U)\right|_{3}=3^{3}$ and so $D=C_{J}(U)$ is a Sylow 3-subgroup of $C_{M}(U)$. Since $Z \leq D$, we have $C_{G}(D)=C_{M}(D)=J U$ which is 3 -closed. Therefore, $N_{G}(D) \leq N_{G}(J)$. Since $r_{1}$ and $r_{2}$ act as transpositions on $\mathcal{T},\left|N_{F}(D U) / J\right|=32$ and so we deduce that $D \in$ $\operatorname{Syl}_{3}\left(C_{G}(U)\right)$. Let $P=N_{N_{G}(U)}(D)$. Then, by the Frattini argument, $P C_{G}(U)=N_{G}(U)$. Therefore, if $N_{G}(U) / C_{G}(U) \cong \operatorname{Sym}(3)$, then $r_{2}$ and $r_{1} r_{2}$ are conjugate in $P$. But $P \leq N_{G}(J), r_{2} \in F \backslash F^{\prime}$ and $r_{1} r_{2} \in F^{\prime}$, which is a contradiction. Hence $N_{G}(U) / C_{G}(U) ¥$ $\operatorname{Sym}(3)$.

\section{Another 3-local subgroup and a 2-local subgroup in the centralizer of an involution}

In this section we study the normalizer of $\left\langle\tau_{5}, \rho\right\rangle$ and construct a 2-local subgroup of $C_{G}\left(r_{2}\right)$.

LeMma 5.1. $И_{G}\left(J_{0}, 3^{\prime}\right)=\{1\}$.

Proof. Suppose that $1 \neq Y \in U_{G}\left(J_{0}, 3^{\prime}\right)$. As every hyperplane of $J_{0}$ contains a conjugate of $Z$ by Lemma 4.10, and by coprime action $Y$ is generated by centralizers of hyperplanes of $J_{0}$, we may assume that $X=C_{Y}(Z) \neq 1$. So $X \in U_{M}\left(J_{0}, 3^{\prime}\right)$. As $X$ is normalized by $A=J_{0} \cap Q$ and $X$ normalizes $Q$,

$$
[A, X] \leq Q \cap X=1 .
$$

But then $X$ centralizes a maximal abelian subgroup of $Q$ and consequently $[Q, X]=1$ by Lemma 2.11, which is a contradiction. Thus $\Lambda_{G}\left(J_{0}, 3^{\prime}\right)=\{1\}$.

Lemma 5.2. Assume that $J=J_{0}$. Then $C_{G}(\rho) \nRightarrow\langle\rho\rangle \times \operatorname{Sp}_{6}(2)$.

Proof. Suppose that $C_{G}(\rho) \cong\langle\rho\rangle \times \operatorname{Sp}_{6}(2)$. Set $E=E\left(C_{G}(\rho)\right)$. Then $E \cong \operatorname{Sp}_{6}(2)$. We have that $r_{2}$ inverts $\rho$ and centralizes $J /\langle\rho\rangle$, so as $J \cap E$ has order $3^{3}$ and $C_{E}(J \cap E)=$ $J \cap E, r_{2}$ induces the trivial automorphism on $E$. Hence $N_{G}(\langle\rho\rangle) \cong \operatorname{Sym}(3) \times E$ and $\left[E, r_{2}\right]=1$. In $E \cap J$ there is an element $\widetilde{\rho}$ with $N_{E}(\langle\widetilde{\rho}\rangle) \cong \mathrm{Sp}_{2}(2) \times \mathrm{Sp}_{4}(2)$ (see [3, p. 46]). Hence $N_{N_{G}(J)}(\langle\rho\rangle) \cap N_{N_{G}(J)}(\langle\widetilde{\rho}\rangle)$ contains a Sylow 2-subgroup $T$ of $N_{G}(J)$. 
Now $\langle\rho, \widetilde{\rho}\rangle=C_{J}(i)$, where $i \in T^{\prime} \leq F^{\prime}$. Since $\mathrm{O}_{2}^{-}(3) \cong \operatorname{Dih}(8)$ and $N_{G}(J) / N_{G}(J)^{\prime} \cong$ $2 \times 2$, we see that the involutions in $N_{G}(J)^{\prime} / J$ invert a --space and centralize a +space with respect to the form given in Theorem 4.5(i). In particular $C_{J}(i)$ is a + -space and so $i$ centralizes a conjugate of $Z$. Hence $\langle\rho, \widetilde{\rho}\rangle$ contains a conjugate of $Z$. But $C_{E}(\langle\rho, \widetilde{\rho}\rangle)^{(\infty)} \cong \operatorname{Sp}_{4}(2)^{(\infty)} \cong \operatorname{Alt}(6)$ contradicts the fact that $M$ is soluble.

Lemma 5.3. Let $B$ be a maximal subgroup of $\left\langle\tau_{5}, \rho, Z\right\rangle$ and assume that $C_{G}(B) \not \leq M$. Then $B \in\left\langle\tau_{5}, \rho\right\rangle^{Q_{2}}$ and either:

(i) $J>J_{0}$ and $C_{G}(B) \cong B \times \mathrm{SU}_{4}(2)$; or

(ii) $J=J_{0}$ and $C_{G}(\rho) \cong\langle\rho\rangle \times \operatorname{Aut}\left(\mathrm{SU}_{4}(2)\right)$.

Proof. Set $U=\left\langle Z, \tau_{5}, \rho\right\rangle$, let $B$ be a maximal subgroup of $U, X=C_{G}(B)$ and $\widetilde{X}=X / B$. Assume that $X \not \leq M$. By Lemma 4.14, $Z$ is the unique conjugate of $Z$ in $U$ and so, as $C_{G}(B) \not \leq M, U=Z B$ and $\widetilde{N_{X}(Z)}=N_{\widetilde{X}}(\widetilde{Z})$.

Assume that $J>J_{0}$. Then, by Lemma 4.13(ii), $N_{X}(Z)=X \cap M=J Q_{1} R_{1}$ and so $N_{\widetilde{X}}(\widetilde{Z})=\widetilde{N_{X}(Z)}=\widetilde{J R_{1} Q_{1}} \cong 3_{+}^{1+2} \cdot \mathrm{SL}_{2}(3)$ which is isomorphic to the centralizer of a 3-central element in $\mathrm{SU}_{4}(2) \cong \mathrm{PSp}_{4}(3)$ by Lemma 2.8. As $B \cap\langle\rho, Z\rangle \neq 1$, we may assume that $\rho \in B$. Then, by Lemma 4.13(i), $C_{M}(B) \leq C_{M}(\rho)=J Q_{1} R_{1}\left\langle r_{2} t_{1}\right\rangle$. As $\left|\left[U, r_{2} t_{1}\right]\right|=9$, we get $C_{M}(B) \leq J Q_{1} R_{1}$ and so $z \in Z^{\#}$ is not $X$-conjugate to its inverse by Lemma 4.13. Thus, as $U_{G}\left(J_{0}, 3^{\prime}\right)=\{1\}$ by Lemma 5.1 and $C_{G}(B) \not \subset M$, we may apply Hayden's Theorem 2.6 to get that $\widetilde{X} \cong \mathrm{SU}_{4}(2)$. Finally, as $J Q_{1}$, splits over $B, X$ splits over $B$ by Gaschütz's theorem [7, Theorem 9.26]. Hence $X$ has the structure described in (i).

Assume that $J=J_{0}$. In this case $B$ is $Q_{2}$-conjugate to $\langle\rho\rangle$. By Lemma 4.13(i), $C_{X}(Z)=X \cap M=J Q_{1} R_{1}$, as $r_{2} t_{1}$ inverts $Z$ and so $\widetilde{C_{X}(Z)}$ is isomorphic to the centralizer of a 3-central element in $\mathrm{SU}_{4}(2)$. Since $r_{2} t_{1}$ inverts $z$, we may use Prince's Theorem 2.7 to obtain $\widetilde{X} \cong \operatorname{Aut}\left(\mathrm{SU}_{4}(2)\right)$ or $\operatorname{Sp}_{6}(2)$. Again Gaschütz's theorem implies that $X \cong\langle\rho\rangle \times E$ where $E \cong \widetilde{X}$. Therefore, by Lemma 5.2, $X$ has the structure claimed in (ii).

Now we consider the possibilities for $B$ when $J>J_{0}$. We have $B \leq U$ and $C_{G}(B) \not \leq$ $M$. Thus, by (i), $C_{G}(B) \cong B \times E$ where $E \cong \mathrm{SU}_{4}(2)$. Consequently, $N_{C_{G}(B)}(J) \cong 3^{2} \times$ $\left(3^{3}: \operatorname{Sym}(4)\right)$. Since $N_{C_{G}(B)}(J) \geq Q_{1}$ and there are exactly three subgroups isomorphic to Alt(4) which contain a given 3-cycle in $\operatorname{Sym}(6)$, we see that $B$ is $Q_{2}$-conjugate to $\left\langle\tau_{5}, \rho\right\rangle$ as claimed.

We now set $r=r_{2}$ and define

$$
K=C_{G}(r) .
$$

We will frequently use the following observation.

Lemma 5.4. $C_{J}(r) Q_{1}$ is a Sylow 3-subgroup of $K$.

Proof. Certainly $C_{J}(r) Q_{1} \leq K$ by Lemma 3.3(i). Because $\left[Q_{1}, C_{J}(r), Q_{1}\right]=[A \cap$ $\left.Q_{1}, Q_{1}\right]=Z, Z$ is a characteristic subgroup of $C_{J}(r) Q_{1}$ and so it follows that $N_{K}\left(C_{J}(r) Q_{1}\right) \leq C_{M}(r)$. As $C_{J}(r) Q_{1} \in \operatorname{Syl}_{3}\left(C_{M}(r)\right)$, the lemma holds. 
Define $E=E\left(C_{G}\left(\left\langle\tau_{5}, \rho\right\rangle\right)\right)$. Then $E \cong \mathrm{SU}_{4}(2)$ by Lemma 5.3.

LEMMA 5.5. $E\left\langle t_{1}, \tau_{5} \tau_{6}\right\rangle \leq K$ and $E\left\langle t_{1}\right\rangle \cong \operatorname{Aut}\left(\mathrm{SU}_{4}(2)\right)$.

Proof. We know that $r$ inverts $\rho$ and exchanges $\tau_{5}$ and $\tau_{6}$ (when they are defined). Hence $r$ normalizes $B=\left\langle\tau_{5}, \rho\right\rangle$ and consequently $r$ normalizes $E$. Furthermore, $r$ centralizes $J \cap E$ and since no automorphism of $E$ acts in this way (see [3, p. 26]), $r$ centralizes $E$. Therefore $E \leq K$.

Since $t_{1}$ inverts $J, t_{1}$ normalizes $\left\langle\tau_{5}, \rho\right\rangle$ and therefore $t_{1}$ normalizes $E$. Since $t_{1}$ inverts $J \cap E$ and, by [3, p. 26], no inner automorphism of $\mathrm{SU}_{4}(2)$ inverts an elementary abelian group of order $27, E\left\langle t_{1}\right\rangle \cong \operatorname{Aut}\left(\mathrm{SU}_{4}(2)\right)$.

From Lemmas 4.13 and 5.3, $Q_{1} R_{1} \leq E$. Furthermore, as $W\left(=C_{F}(r)\right)$ normalizes $[J, r]=\langle\rho\rangle$, we have that $C_{W}(\rho) \leq E$. In particular, the following lemma holds.

Lemma 5.6. $\left\langle\tau_{5} \tau_{6}\right\rangle E=\left\langle C_{W}(\rho), Q_{1} R_{1} C_{J}(r)\right\rangle$.

Proof. As $Q_{1} R_{1} C_{J}(r)$ contains the maximal parabolic subgroup of shape $3_{+}^{1+2} \cdot \mathrm{SL}_{2}(3)$ of $E \cong \mathrm{SU}_{4}(2)$, we have that $Y=Q_{1} R_{1} C_{J}(r)$ is a maximal subgroup of $E\left\langle\tau_{5} \tau_{6}\right\rangle$. Since $C_{W}(\rho) \not \leq Y$, the result follows.

When $J>J_{0}$, as $N_{G}(J)$ acts 2-transitively on $\mathcal{T},\left\langle\tau_{5}, \tau_{6}\right\rangle$ is $G$-conjugate to each subgroup $J_{i} \cap J_{j}$ for $1 \leq i<j \leq 4$. When $J=J_{0}$ we have the same result from the construction of $J_{1}, J_{2}, J_{3}$ and $J_{4}$ in Section 4. Hence we may apply Lemma 5.3 to obtain the following conclusion.

Lemma 5.7. Assume that $1 \leq i<j \leq 4$.

(i) If $J>J_{0}$, then $C_{G}\left(J_{i} \cap J_{j}\right) \cong\left(J_{i} \cap J_{j}\right) \times \mathrm{SU}_{4}(2)$.

(ii) If $J=J_{0}$, then $C_{G}\left(J_{i} \cap J_{j}\right) \cong\left(J_{i} \cap J_{j}\right) \times \operatorname{Aut}\left(\mathrm{SU}_{4}(2)\right)$.

For $1 \leq i<j \leq 4$, define

$$
E_{i j}=E\left(C_{G}\left(J_{i} \cap J_{j}\right)\right) .
$$

Lemma 5.8. For $1 \leq i<j \leq 4$ and $k \in\{i, j\}, E_{i j} \cap J_{k}$ is conjugate to $Z$ and is 3 -central in $E_{i j}$. In particular, $C_{G}\left(J_{i}\right) \cong\left(J_{i} \cap J_{j}\right) \times 3_{+}^{1+2}: \mathrm{SL}_{2}(3)$ if $J>J_{0}$, and $C_{G}\left(J_{i}\right) \cong\left(J_{i} \cap\right.$ $\left.J_{j}\right) \times 3_{+}^{1+2}: \mathrm{SL}_{2}(3) \cdot 2$ if $J=J_{0}$.

Proof. Let $1 \leq i \leq 4$. By Lemma 4.11, $J_{i}$ is normalized by a Sylow 3-subgroup $T_{i}$ of $N_{G}(J)$ and $C_{T_{i}}\left(J_{i}\right)$ has index 3 in $T_{i}$. In particular, as $\left|C_{G}\left(J_{i} \cap J_{j}\right)\right|_{3}=3|J|$, $C_{T_{i}}\left(J_{i}\right) \in \operatorname{Syl}_{3}\left(C_{G}\left(J_{i} \cap J_{j}\right)\right)$. Therefore $J_{i} \cap E_{i j}$ is normalized by a Sylow 3-subgroup of $E_{i j}$. Because $\left|J_{i} \cap E_{i j}\right|=3, J_{i} \cap E_{i j}$ is 3-central in $E_{i j}$ as $J_{i}$ is normal in $T_{i}$, and so this subgroup is also normal in a Sylow 3-subgroup of $G$.

Define

$$
\Sigma=\left\langle O_{2}\left(C_{K}\left(J_{k}\right)\right) \mid 1 \leq k \leq 4\right\rangle .
$$

In the next lemma we use the fact that if $x \in X \cong \mathrm{SU}_{4}(2)$ is an involution which centralizes a subgroup of order nine, then $x$ is 2-central and

$$
C_{X}(x) \cong 2_{+}^{1+4} \cdot(3 \times \operatorname{Sym}(3)) \cong\left(\mathrm{SL}_{2}(3) \circ \mathrm{SL}_{2}(3)\right) \cdot 2
$$


where $\circ$ denotes a central product (see [3, p. 26]).

Lemma 5.9. Assume that $1 \leq i<j \leq 4$.

$$
\begin{array}{r}
O_{2}\left(C_{K}\left(J_{i}\right)\right) \cong O_{2}\left(C_{K}\left(J_{j}\right)\right) \cong \mathrm{Q}_{8},\left[O_{2}\left(C_{K}\left(J_{i}\right)\right), O_{2}\left(C_{K}\left(J_{j}\right)\right)\right]=1 \text { and } \\
O_{2}\left(C_{K}\left(J_{i} \cap J_{j}\right)\right)=O_{2}\left(C_{K}\left(J_{i}\right)\right) O_{2}\left(C_{K}\left(J_{j}\right)\right) \cong 2_{+}^{1+4} .
\end{array}
$$

(ii) $\quad \Sigma$ is extraspecial of +-type and order $2^{9}$.

Proof. Suppose that $1 \leq i<j \leq 4$. Then $J_{i} \leq C_{G}(r)$ by Lemma 5.7. If $J>J_{0}$, then $r \in E_{i j}$ by Lemma 5.3. If $J=J_{0}$, then, as $\left[J_{1}, R_{2}\right]=1$ and $r \in Z\left(R_{2}\right) \leq C_{G}\left(J_{1}\right)^{\prime}$, we have $r \in E_{12}$ and consequently $r \in E_{i j}$ as $W$ acts 2-transitively on $\left\{J_{1}, J_{2}, J_{3}, J_{4}\right\}$.

Since $r \in E_{i j}$ and $\left|C_{J}(r) \cap E_{i j}\right|_{3} \geq 9, r$ is a 2-central involution in $E_{i j}$. It follows that $K \cap E_{i j}$ has shape $2_{+}^{1+4} \cdot(3 \times \operatorname{Sym}(3))$ and, in particular, $O_{2}\left(C_{K}\left(J_{i} \cap J_{j}\right)\right) \cong 2_{+}^{1+4}$. Furthermore, as $J_{i} \cap E_{i j}$ is 3-central by Lemma 5.8, we get $O_{2}\left(C_{K}\left(J_{i}\right)\right) \cong \mathrm{Q}_{8}$ and $O_{2}\left(C_{K}\left(J_{i} \cap J_{j}\right)\right)=O_{2}\left(C_{K}\left(J_{i}\right)\right) O_{2}\left(C_{K}\left(J_{j}\right)\right)$. Since $O_{2}\left(C_{K}\left(J_{i} \cap J_{j}\right)\right)$ contains exactly two subgroups isomorphic to $\mathrm{Q}_{8},\left[O_{2}\left(C_{K}\left(J_{i}\right)\right), O_{2}\left(C_{K}\left(J_{j}\right)\right)\right]=1$. This completes the proof of (i).

Part (i) shows that $\Sigma$ is isomorphic to a central product of four quaternion groups of order eight. Hence $\Sigma$ is extraspecial of +-type and order $2^{9}$. So (ii) holds.

Recall from Corollaries 4.6 and 4.7 that

$$
t_{2} \in N_{G}(S) \leq M \cap N_{G}(J)
$$

and $R_{1}^{t_{2}}=R_{2}$.

Lemma 5.10. $J_{1}$ is centralized by $R_{2}, R_{2} \leq \Sigma$ and $R_{2}=C_{\Sigma}(Z)$.

Proof. Suppose first that $J=J_{0}$. Then $J_{1}=C_{A}(r) \leq Q_{1}=C_{Q}\left(R_{2}\right)$ by Lemma 3.3(i). Thus $\left[J_{1}, R_{2}\right]=1$ and so $R_{2}=O_{2}\left(C_{K}\left(J_{1}\right)\right) \leq \Sigma$.

Now suppose that $J>J_{0}$. We have that $\tau_{1}$ commutes with $Q_{1}$ and $\left[\left\langle\tau_{5}, \tau_{6}\right\rangle, Q_{1}\right]=1$ by Lemma 4.13. Hence

$$
C_{J}\left(Q_{1}\right)=\left\langle\tau_{1}, \tau_{5}, \tau_{6}\right\rangle=\left\langle\tau_{5}, A \cap Q_{2}\right\rangle .
$$

Thus $C_{J}\left(Q_{2}\right)=C_{J}\left(Q_{1}\right)^{t_{2}}=\left\langle\tau_{2}, \tau_{3}, \tau_{4}\right\rangle=\left\langle\tau_{2}, A \cap Q_{1}\right\rangle$. By Lemma 4.8, $C_{J}\left(Q_{1}\right)$ is centralized by $R_{1}$. Therefore $J_{1}=\left\langle\tau_{2}, \tau_{3}, \tau_{4}\right\rangle$ is centralized by $R_{2}=R_{1}^{t_{2}}$. Hence $R_{2}=O_{2}\left(C_{K}\left(J_{1}\right)\right) \leq \Sigma$.

Since $R_{2}$ commutes with $Z, R_{2} \leq C_{\Sigma}(Z)$ and, as $C_{\Sigma}(Z)$ is extraspecial, $R_{2}=C_{\Sigma}(Z)$ from the structure of $M$.

LeMma 5.11. $W\left\langle t_{1}\right\rangle \leq N_{K}(\Sigma)$.

Proof. Since $W\left\langle t_{1}\right\rangle$ permutes $\left\{J_{1}, J_{2}, J_{3}, J_{4}\right\}$ and is contained in $K, W\left\langle t_{1}\right\rangle \leq N_{K}(\Sigma)$ by the definition of $\Sigma$.

Lemma 5.12. $W=N_{K}\left(C_{J}(r)\right)=N_{N_{K}(\Sigma)}\left(C_{J}(r)\right)$. In particular, $N_{N_{K}(\Sigma)}\left(C_{J}(r)\right)$ controls $K$-fusion in $C_{J}(r)$. 
Proof. $C_{G}\left(C_{J}(r)\right)=C_{M}\left(C_{J}(r)\right)=J\langle r\rangle$. Hence $J$ is normal in $N_{G}\left(C_{J}(r)\right)$ and thus $W=$ $N_{K}\left(C_{J}(r)\right)$. By Lemma 5.11, $W \leq N_{K}(\Sigma)$ and so $N_{K}\left(C_{J}(r)\right)=N_{N_{K}(\Sigma)}\left(C_{J}(r)\right)$. Further, by Lemma 4.1, $N_{G}(J)$ controls fusion in $J$ and so $N_{K}\left(C_{J}(r)\right)$ controls $K$-fusion in $C_{J}(r)$. As $N_{K}\left(C_{J}(r)\right)=N_{N_{K}(\Sigma)}\left(C_{J}(r)\right)$, this fusion takes place in $N_{K}(\Sigma)$.

Lemma 5.13. Every $J_{1}$-signalizer in $K$ is contained in $\Sigma$. In particular, $N_{K}\left(J_{1}\right) \leq$ $N_{K}(\Sigma)$

Proof. Let $\Sigma_{1} \leq K$ be a $J_{1}$-signalizer. Let $X_{1}$ be a hyperplane in $J_{1}$ such that $C_{G}\left(X_{1}\right) \leq$ $M$. Then $C_{\Sigma_{1}}\left(X_{1}\right) \leq M$ is normalized by $J_{1}$ and so $C_{\Sigma_{1}}\left(X_{1}\right) \leq R_{2} \leq \Sigma$ by Lemma 5.10. In particular, $\left[C_{\Sigma_{1}}(Z), J_{1}\right]=1$.

Suppose next that $X_{1}$ is a hyperplane such that $C_{G}\left(X_{1}\right) \not \geq M$. Then, by Lemma 5.3, we may assume that $X_{1}=J_{1} \cap J_{2}$. Since $r$ is 2-central in $E_{12}, O_{2}\left(C_{K}\left(J_{1} \cap J_{2}\right)\right)$ is the unique maximal $J_{1}$-signalizer in $C_{G}\left(X_{1}\right)$. Hence, by Lemma 5.9(i), $C_{\Sigma_{1}}\left(X_{1}\right) \leq \Sigma$ in this case as well. Because

$$
\Sigma_{1}=\left\langle C_{\Sigma_{1}}\left(X_{1}\right)|| J_{1}: X_{1} \mid \leq 3\right\rangle \leq \Sigma,
$$

we have that every $J_{1}$-signalizer is contained in $\Sigma$. Thus $\Sigma$ is the unique maximal member of $U_{K}\left(J_{1}, 3^{\prime}\right)$ and so $N_{K}\left(J_{1}\right) \leq N_{K}(\Sigma)$ as $N_{K}\left(J_{1}\right)$ acts via conjugation on the maximal elements of $U_{K}\left(J_{1}, 3^{\prime}\right)$.

Lemma 5.14. $C_{K}(\Sigma)=\langle r\rangle$.

Proof. If $C_{K}(\Sigma)$ is a $3^{\prime}$-group, then $C_{K}(\Sigma)$ is normalized by $J_{1}$ and so $C_{K}(\Sigma) \leq Z(\Sigma)=$ $\langle r\rangle$ by Lemma 5.13. So suppose that $C_{K}(\Sigma)$ has order divisible by 3 . Since $C_{J}(r) Q_{1} \in$ $\operatorname{Syl}_{3}(K)$ by Lemma 5.4, and $C_{J}(r) Q_{1} \leq W \leq N_{K}(\Sigma)$ by Lemma 5.11, $C_{J}(r) Q_{1} \cap C_{G}(\Sigma)$ is a Sylow 3-subgroup of $C_{G}(\Sigma)$. As $Z$ does not centralize $\Sigma$, we have $C_{J}(r) Q_{1} \cap$ $C_{G}(\Sigma) \leq C_{J}(r)$. Now, for $1 \leq i<j \leq 4$,

$$
C_{C_{J}(r)}\left(O_{2}\left(C_{K}\left(J_{i} \cap J_{j}\right)\right)\right)=J_{i} \cap J_{j},
$$

and consequently $C_{C_{J}(r)}(\Sigma) \leq J_{1} \cap J_{2} \cap J_{3} \cap J_{4}=1$, which is a contradiction.

Lemma 5.15. $\Sigma /\langle r\rangle$ is a minimal normal subgroup of $N_{K}(\Sigma) /\langle r\rangle$.

Proof. Suppose that $U \leq \Sigma$ and $U /\langle r\rangle$ is a minimal normal subgroup of $N_{K}(\Sigma) /\langle r\rangle$ of minimal order. Aiming for a contradiction, assume that $U \neq \Sigma$. Then either $|\Sigma: U| \leq 2^{4}$ or $|U /\langle r\rangle| \leq 2^{4}$. In particular, as $Q_{1}$ normalizes $\Sigma$ (see Lemma 5.13) and $\mathrm{GL}_{4}(2)$ has elementary abelian Sylow 3 -subgroups, $Z$ centralizes one of $U$ or $\Sigma / U$. By Lemma 5.10, either $U \leq R_{2}$ or $|\Sigma: U| \leq 2^{2}$ and $U \geq[\Sigma, Z]$.

Since $C_{J}(r)$ acts nontrivially on $R_{2}$, we get $U=R_{2}$ or $U=[\Sigma, Z]$. In the latter case, $U_{1}=C_{\Sigma}(U)$ is normalized by $N_{K}(\Sigma)$ and has order smaller than $U$. Hence the minimal choice of $U$ implies that $U=R_{2}$. However, $W \leq N_{G}(\Sigma)$ by Lemma 5.11 and $W$ does not normalize $R_{2}$, and so we have a contradiction. 
THEOREM 5.16. One of the following results holds.

(i) $J=J_{0}$ and $N_{G}(\Sigma) / \Sigma \cong \operatorname{Aut}\left(\mathrm{SU}_{4}(2)\right)$ or $\mathrm{Sp}_{6}(2)$; or

(ii) $J>J_{0}$ and $N_{G}(\Sigma) / \Sigma \cong\left(3 \times \mathrm{SU}_{4}(2)\right): 2$.

Furthermore, $E\left\langle\tau_{5} \tau_{6}, t_{1}\right\rangle \leq N_{K}(\Sigma)$ and $\Sigma /\langle r\rangle$ is isomorphic to the natural $E \Sigma / \Sigma$ module.

Proof. From Lemma 5.11, $W\left\langle t_{1}\right\rangle \leq N_{G}(\Sigma)$. Set $L=J_{1} Q_{1}$. Then $L \leq W$ and so $L \leq$ $N_{G}(\Sigma)$. By Lemma 5.13, $\Sigma$ is a maximal signalizer in $K$ for $L$ and for $C_{J}(r)$. Hence $N_{K}(L)$ and $N_{K}\left(C_{J}(r)\right)$ both normalize $\Sigma$.

Suppose that $J=J_{0}$. Then $J_{1} Q_{1}=\left(A \cap Q_{1}\right) Q_{1} \leq Q_{1}$ and so $R_{1} \leq N_{K}\left(Q_{1}\right) \leq N_{K}(\Sigma)$. Therefore Lemma 5.6 implies that $\left\langle E, t_{1}\right\rangle \leq N_{K}(\Sigma)$. In particular, the quotient $C_{N_{K}(\Sigma) / \Sigma}(Z \Sigma / \Sigma)$ is isomorphic to the centralizer of a 3-element in $\mathrm{SU}_{4}(2)$ and is inverted by $t_{1} \Sigma$. Hence Theorem 2.7 shows that (i) holds.

Suppose that $J>J_{0}$. This time $N_{K}\left(J_{1} Q_{1}\right)$ does not contain $R_{1}$. On the other hand, $N_{K}(\Sigma) \geq N_{K}\left(C_{J}(r)\right) \Sigma=W \Sigma$ and $W \Sigma / \Sigma$ has shape $3^{4}:(\operatorname{Sym}(4) \times 2)$. By the Frattini argument, $N_{N_{K}(\Sigma) / \Sigma}\left(C_{J}(r) \Sigma / \Sigma\right)=N_{N_{K}(\Sigma)}\left(C_{J}(r)\right)$. Since $N_{K}\left(C_{J}(r)\right)=W$, we now have $N_{N_{K}(\Sigma) / \Sigma}\left(C_{J}(r) \Sigma / \Sigma\right)=W \Sigma / \Sigma$.

Since $C_{G}(\Sigma)=\langle r\rangle$ by Lemma $5.14, N_{K}(\Sigma) / \Sigma$ is isomorphic to a subgroup of $\mathrm{O}_{8}^{+}(2)$. Because $N_{N_{K}(\Sigma) / \Sigma}\left(C_{J}(r) \Sigma / \Sigma\right)=W \Sigma / \Sigma$, we infer from the list of maximal subgroups of $\mathrm{O}_{8}^{+}(2)$ given in [3, p. 85] that either $N_{K}(\Sigma)=W \Sigma$ or $N_{K}(\Sigma) / \Sigma \cong\left(3 \times \mathrm{SU}_{4}(2)\right): 2$. In the latter case we have (ii) so suppose that $N_{K}(\Sigma)=W \Sigma$. Let $T \in \operatorname{Syl}_{2}\left(N_{K}(\Sigma)\right.$ ). We claim that $T \in \operatorname{Syl}_{2}(K)$. Assume that $x \in N_{K}(T) \backslash N_{K}(\Sigma)$. Then, as $\Sigma^{x} \neq \Sigma, J(T /\langle r\rangle) \not \Sigma \Sigma /\langle r\rangle$. Hence, setting $H=\left\langle J(T)^{N_{K}(\Sigma)}\right\rangle$ and noting that $\left|O_{3}\left(N_{K}(\Sigma) / \Sigma\right)\right|=3^{4}$, we may apply [1, (32.5)] to get that $H / \Sigma$ is a direct product of four subgroups isomorphic to $\mathrm{SL}_{2}(2)$. But then the 2-rank of $W / \Sigma$ is at least four, contrary to $T / \Sigma \cong \operatorname{Dih}(8) \times 2$. Hence $N_{K}(T) \leq N_{K}(\Sigma)$ and, in particular, $T \in \operatorname{Syl}_{2}(K)$.

From Lemma 5.5, $E \leq K$. Since $T \in \operatorname{Syl}_{2}(K), T / \Sigma \cong \operatorname{Dih}(8) \times 2$ and $E$ contains an extraspecial subgroup of order $2^{5}$ with centre $\left\langle r_{1}\right\rangle$, we have that $r_{1}$ is $K$-conjugate to an element of $\Sigma$. Thus there is some $x \in K$ such that $\left\langle r_{1}, r\right\rangle \leq \Sigma^{x}$. Since $r_{1}^{t_{2}}=r$ and since $r_{1}$ and $r r_{1}$ are $\Sigma^{x}$-conjugate, $N_{G}\left(\left\langle r_{1}, r\right\rangle\right) / C_{G}\left(\left\langle r_{1}, r\right\rangle\right) \cong \operatorname{Sym}(3)$. This contradicts Lemma 4.15. Hence (ii) holds.

We have already seen that $E \leq N_{K}(\Sigma)$ if $J=J_{0}$. If $J>J_{0}$, then $N_{N_{K}(\Sigma)}(Z)$ contains a subgroup $\left(3 \times 3_{+}^{1+2}\right) \cdot \mathrm{SL}_{2}(3) \cdot 2$. Since $N_{K}(Z)=C_{M}(r)=Q_{1} R_{1} R_{2} C_{J}(r)\left\langle t_{1}\right\rangle$, we have $C_{M}(r) \leq N_{K}(\Sigma)$. Now $E\left\langle\tau_{5} \tau_{6}, t_{1}\right\rangle \leq N_{K}(\Sigma)$ by Lemma 5.6. Finally, as $E$ acts irreducibly on $\Sigma /\langle r\rangle$ by Lemma $5.15, \Sigma /\langle r\rangle$ is the natural $E$-module.

We need just two final details before we can move on to determine the structure of $K$.

Lemma 5.17. The following properties hold:

(i) $\quad N_{K}(Z) \leq N_{K}(\Sigma)$; and

(ii) $\quad N_{K}\left(J_{i} \cap J_{j}\right) \leq N_{K}(\Sigma)$, for $1 \leq i<j \leq 4$.

Proof. For (i) we note that $N_{K}(Z)=C_{M}(r) \leq E\left\langle\tau_{5} \tau_{6}, t_{1}\right\rangle \Sigma \leq N_{K}(\Sigma)$ by Theorem 5.16. 
By Lemma 5.9(i), $O_{2}\left(C_{K}\left(J_{i} \cap J_{j}\right)\right) \leq \Sigma$ and, as $r$ is a 2-central element in $E_{i j}$, $C_{J}(r) \in \operatorname{Syl}_{3}\left(C_{K}\left(J_{i} \cap J_{j}\right)\right)$. Hence

$$
N_{K}\left(J_{i} \cap J_{j}\right)=N_{N_{K}\left(J_{i} \cap J_{j}\right)}\left(C_{J}(r)\right) O_{2}\left(C_{K}\left(J_{i} \cap J_{j}\right)\right) \leq N_{K}(\Sigma)
$$

by Lemma 5.13 .

\section{The structure of $K$}

In this section we prove Theorem 6.11, which asserts that $K=N_{K}(\Sigma)$. We retain the notation introduced in the previous sections. We further set $K_{1}=N_{K}(\Sigma)$ and denote by the natural homomorphism from $K$ onto $K /\langle r\rangle$.

By Lemma 5.15 , the subgroup $\widetilde{\Sigma}$ can be regarded as the eight-dimensional irreducible GF(2)-module for $\bar{K}_{1} / \widetilde{\Sigma}$. Thus we may employ Proposition 2.12 to obtain information about various centralizers of elements of order two and three in $\widetilde{\Sigma}$. Using Proposition 2.12(ii), $\widetilde{K}_{1}$ has two orbits on $\widetilde{\Sigma}$. We pick representatives $\widetilde{x}=x\langle r\rangle$ and $\widetilde{y}=y\langle r\rangle$ of these orbits, with $\widetilde{x}$ singular and $\widetilde{y}$ nonsingular. It follows that $x$ is an involution and $y$ has order four.

Our aim is to show that $\widetilde{\Sigma}$ is strongly closed in $\widetilde{K}$ and then to use Goldschmidt's theorem [5] to show that $K=K_{1}$.

LeMma 6.1. $\widetilde{K}_{1}$ contains a Sylow 2-subgroup of $C_{\widetilde{K}}(\widetilde{y})$. In particular:

(i) if $E\left(\widetilde{K}_{1} / \widetilde{\Sigma}\right) \cong \mathrm{SU}_{4}(2)$, then $\left|C_{\widetilde{K}}(\widetilde{y})\right|_{2}=2^{12}$; and

(ii) if $\widetilde{K}_{1} / \widetilde{\Sigma} \cong \operatorname{Sp}_{6}(2)$, then $\left|C_{\widetilde{K}}(\widetilde{y})\right|_{2}=2^{14}$.

Proof. Let $T$ be a Sylow 2-subgroup of $C_{\widetilde{K}_{1}}(\widetilde{y})$ and assume that $T_{1}$ is a 2-group with $\left|T_{1}: T\right|=2$. Choose $u \in T_{1} \backslash T$. If $\left|\widetilde{\Sigma^{u}} \widetilde{\Sigma} / \widetilde{\Sigma}\right| \leq 2$, then $\left|\widetilde{\Sigma}^{u} \cap \widetilde{\Sigma}\right| \geq 2^{7}$. But by Proposition 2.12(iv), $\widetilde{K_{1}}$ has no 2-elements not in $\widetilde{\Sigma}$ which centralize a subgroup of index two in $\widetilde{\Sigma}$. Therefore $\widetilde{\Sigma}=\widetilde{\Sigma}^{u}$ and so $u \in T_{1} \cap \widetilde{K}_{1}=T$, which is a contradiction. Hence $\left|\widetilde{\Sigma^{n}} \widetilde{\Sigma} / \widetilde{\Sigma}\right| \geq 4$.

If $E\left(\widetilde{K}_{1} / \widetilde{\Sigma}\right) \cong \mathrm{SU}_{4}(2)$, then $T / \widetilde{\Sigma}$ is a semidihedral group of order $2^{4}$ by Proposition 2.12(ii). Since $\widetilde{\Sigma^{u}} \widetilde{\Sigma} / \widetilde{\Sigma}$ is a normal elementary abelian subgroup of $T / \widetilde{\Sigma}$ of order at least 4 , we have a contradiction. Hence $\widetilde{K}_{1} / \bar{\Sigma} \cong \mathrm{Sp}_{6}(2)$ by Lemma 5.16. Now Proposition 2.12(ii) gives

$$
C_{\widetilde{K}_{1}}(\widetilde{y}) / \widetilde{\Sigma} \cong \mathrm{G}_{2}(2) .
$$

As, by [8, Table 3.3.1], $\mathrm{G}_{2}(2)$ does not contain elementary abelian subgroups of order $2^{4}, 2^{6} \geq\left|\widetilde{\Sigma}^{u} \cap \widetilde{\Sigma}\right| \geq 2^{5}$. But then all involutions in $\widetilde{\Sigma}^{u}$ centralize a subgroup of order at least $2^{5}$ in $\widetilde{\Sigma}$, and so Proposition 2.12(i) and (iv) show that all the involutions in $\widetilde{\Sigma} u \widetilde{\Sigma} / \widetilde{\Sigma}$ are unitary transvections and are conjugate in $\widetilde{K_{1}} / \widetilde{\Sigma}$. Since the two classes of involutions in $C_{\widetilde{K}_{1}}(\widetilde{y}) / \widetilde{\Sigma} \cong \mathrm{G}_{2}(2)$ are not fused in $\widetilde{K_{1}} / \widetilde{\Sigma}$, we infer that

$$
\widetilde{\Sigma} \widetilde{\Sigma}^{u} / \widetilde{\Sigma} \leq\left(C_{\widetilde{K}_{1}}(\widetilde{y}) / \widetilde{\Sigma}\right)^{\prime} \cong \mathrm{G}_{2}(2)^{\prime} \cong \mathrm{SU}_{3}(3) .
$$


Since, by [8, Table 3.3.1], $\mathrm{SU}_{3}(3)$ has no elementary abelian groups of order eight, $|\widetilde{\Sigma} u \widetilde{\Sigma} / \widetilde{\Sigma}|=4$. This means that $\left|\widetilde{\Sigma}^{u} \cap \widetilde{\Sigma}\right|=2^{6}$ and consequently all the involutions in $\widetilde{\Sigma}^{u} \widetilde{\Sigma} / \widetilde{\Sigma}$ have the same centralizer in $\widetilde{\Sigma}$. As centralizers of involutions in $\mathrm{G}_{2}(2)^{\prime}$ are maximal subgroups [3, p. 14], we conclude that $\widetilde{\Sigma}^{u} \cap \widetilde{\Sigma}$ is normalized by $\left(C_{\widetilde{K}_{1}}(\widetilde{y}) / \widetilde{\Sigma}\right)^{\prime}$. Thus $\left(C_{\widetilde{K}_{1}}(\widetilde{y}) / \widetilde{\Sigma}\right)^{\prime}$ centralizes $\widetilde{\Sigma}$, which is impossible. This contradiction proves the lemma. The order of $T$ is calculated using Proposition 2.12(iii).

Lemma 6.2. Let $S_{1}$ be a Sylow 3-subgroup of $C_{\widetilde{K}_{1}}(\widetilde{x})$ or $C_{\widetilde{K}_{1}}(\widetilde{y})$. Then $N_{\widetilde{K}}\left(S_{1}\right) \leq \widetilde{K}_{1}$. In particular, for $z \in \widetilde{\Sigma}^{\#}, C_{\widetilde{K}_{1}}(z)$ contains a Sylow 3-subgroup of $C_{\widetilde{K}}(z)$.

Proof. We consider $\tilde{y}$ first. By Proposition 2.12(iii), $S_{1}$ has centre of order three and, as faithful $\mathrm{GF}(2)$-representations of extraspecial groups of type $3_{+}^{1+2}$ have dimension six, $\left|C_{\widetilde{\Sigma}}\left(Z\left(S_{1}\right)\right)\right|=4$. As $\left|C_{\widetilde{\Sigma}}(Z)\right|=4$ we may assume, using Proposition 2.12 (iii), that $Z=Z\left(S_{1}\right)$. On the other hand, Lemma 5.17(i) gives $C_{M}(r) \leq K_{1}$. Hence $N_{\widetilde{K}}\left(S_{1}\right) \leq \widetilde{K}_{1}$.

Now we consider $\widetilde{x}$. By Lemma 5.9(i), $O_{2}\left(C_{K}\left(J_{1} \cap J_{2}\right)\right) \leq \Sigma$ and $O_{2}\left(C_{K}\left(J_{1} \cap J_{2}\right)\right)$ contains noncentral involutions of $\Sigma$. Hence, by comparing the orders of $S_{1}$ and $J_{1} \cap J_{2}$, we may assume that $S_{1}=J_{1} \cap J_{2}$. Then, by Lemma $5.17(\mathrm{ii}), N_{\widetilde{K}}\left(S_{1}\right) \leq \widetilde{K}_{1}$.

Let $\widetilde{E} \leq \widetilde{K}_{1}$ such that $\widetilde{E} / \widetilde{\Sigma}=E\left(\widetilde{K}_{1} / \widetilde{\Sigma}\right)$. Either $\widetilde{E} / \widetilde{\Sigma} \cong \mathrm{SU}_{4}(2)$ or $\mathrm{Sp}_{6}(2)$. By Proposition 2.12(iii) there are exactly three classes of elements of order three in $\widetilde{E}$. As a Sylow 3 -subgroup of $\widetilde{E}$ is isomorphic to the wreath product $3<3$, there is a unique conjugacy class of elementary abelian subgroups of order 27 in $\widetilde{E}$, and these subgroups contain elements from each of the conjugacy classes of elements of order three. As $\widetilde{C_{J}(r)} \cap \widetilde{E}$ is elementary abelian of order 27 , there are representatives of these elements in $\widetilde{C_{J}(r)} \cap \widetilde{E}$. It follows that every element of order three in $\widetilde{K}$ is conjugate to an element of $\widetilde{C_{J}(r)}$. So, using Lemma 5.12, we get the following lemma.

Lemma 6.3. Two elements of order three in $\widetilde{K}_{1}$ are conjugate in $\widetilde{K}$ if and only if they are conjugate in $\widetilde{K}_{1}$.

For $J>J_{0}$, we establish some further notation. Let $\sigma \in \widetilde{K_{1}}$ have order three such that $\sigma \widetilde{\Sigma}$ is centralized by $\widetilde{E} / \widetilde{\Sigma}$. We note that, by Lemma $6.3, \sigma$ is not $\widetilde{K}$-conjugate to any element in $\widetilde{E}$.

Lemma 6.4. Suppose that $\widetilde{u} \in \widetilde{K}_{1} \backslash \widetilde{\Sigma}$ is an involution which is $\widetilde{K}$-conjugate to some involution in $\widetilde{\Sigma}$. Assume that $v \in C_{\widetilde{K}_{1}}(\widetilde{u})$ has order three. Then:

(i) $C_{\widetilde{\Sigma}}(v) \neq 1$;

(ii) $\langle v\rangle$ and $Z$ are not $\widetilde{K}$-conjugate;

(iii) if $J=J_{0}$, then $v$ and $\rho$ are not $\widetilde{K}$-conjugate; and

(iv) $\left|C_{\widetilde{E}}(\widetilde{u})\right|$ is not divisible by 9 .

Proof. Let $\widetilde{a} \in \widetilde{\Sigma}$ and assume that $\widetilde{a}$ and $\widetilde{u}$ are $\widetilde{K}$-conjugate. By Lemma $6.2, \widetilde{K}_{1}$ contains a Sylow 3-subgroup of $C_{\widetilde{K}}(\widetilde{a})$. By Lemma $6.3, v$ is $\widetilde{K}_{1}$-conjugate to an element $\mu$ of $C_{\widetilde{K_{1}}}(\widetilde{a})$. Now obviously $C_{\widetilde{\Sigma}}(\mu) \neq 1$ and so the same holds for $v$, which is (i). 
If $\langle v\rangle$ is $\widetilde{K}$-conjugate to $Z$ or to $\langle\rho\rangle$ in the case where $J=J_{0}$, this conjugation is also achieved in $\widetilde{K}_{1}$ by Lemma 6.3 . Hence we may assume that $\widetilde{a}$ is conjugate to $\widetilde{u}$ in $M \cap K$, or in $N_{K}(\langle\rho\rangle)$ if $J=J_{0}$. Since $M \cap K \leq K_{1}$ and $N_{K}(\langle\rho\rangle)$ when $J=J_{0}$ by Lemma 5.17, we have a contradiction. Hence (ii) and (iii) hold.

Assume now that $S_{1} \leq C_{\widetilde{E}}(\widetilde{u})$ with $\left|S_{1}\right|=9$. Then $S_{1}$ is conjugate to a Sylow 3subgroup $S_{2}$ of $C_{\widetilde{E}}(\widetilde{a})$. So, by Lemma 6.2 and Proposition 2.12(ii), we may assume that $\widetilde{a}=\widetilde{y}$ and thus $S_{2}$ is extraspecial of order 27. Hence $S_{1}$ contains some element which is conjugate to $Z\left(S_{2}\right)$. But $Z\left(S_{2}\right)$ is conjugate to $Z$, and this contradicts (ii). Thus the Sylow 3-subgroups of $C_{\widetilde{E}}(\widetilde{u})$ have order three and so (iii) holds.

Lemma 6.5. Suppose that $\widetilde{u} \in \widetilde{K}_{1} \backslash \widetilde{\Sigma}$ is an involution which is $\widetilde{K}$-conjugate to some involution in $\widetilde{\Sigma}$. Then one of the following results holds.

(i) $\widetilde{u} \in \widetilde{E},|[\widetilde{\Sigma}, \widetilde{u}]|=4$; and

(a) if $E\left(\widetilde{K}_{1} / \widetilde{\Sigma}\right) \cong \mathrm{SU}_{4}(2)$, then $\left|C_{\widetilde{E}}(\widetilde{u})\right|=2^{13}$, and

(b) if $\widetilde{K}_{1} / \widetilde{\Sigma} \cong \operatorname{Sp}_{6}(2)$, then $\left|C_{\widetilde{E}}(\widetilde{u})\right|=2^{15}$.

(ii) $J>J_{0}, \sigma^{\widetilde{u}}=\sigma^{-1}, C_{\widetilde{E} / \widetilde{\Sigma}}(\widetilde{u}) \cong 2 \times \operatorname{Sym}(4) \leq \operatorname{Sym}(6)$, and $|[\widetilde{\Sigma}, \widetilde{u}]|=16$.

Proof. If $|[\widetilde{u}, \widetilde{\Sigma}]|=16$, then all involutions in the coset $\widetilde{\Sigma} \widetilde{u}$ are conjugate by elements of $\widetilde{\Sigma}$. Hence, by Proposition 2.12(i), $\widetilde{u}$ centralizes some nontrivial 3-element $v \in \widetilde{E}$. By Lemma 6.4(i), $C_{\widetilde{\Sigma}}(v) \neq 1$. If $J=J_{0}$, then, by Proposition 2.12(iii), $\langle v\rangle$ is conjugate to $Z$ or $\langle\rho\rangle$, which contradicts Lemma 6.4(ii) and (iii). So assume that $J>J_{0}$. If $\widetilde{u} \notin \widetilde{E}$, we obtain assertion (ii) from Proposition 2.12(i) and Lemma 6.4(iv). So assume that $\widetilde{u} \in \widetilde{E}$. Then $C_{\widetilde{E} / \widetilde{\Sigma}}(\widetilde{u})$ is contained in a parabolic subgroup of $\widetilde{E} / \widetilde{\Sigma}$ of shape $2^{4}: \operatorname{Alt}(5)$ and so $v$ acts fixed point freely on $\widetilde{\Sigma}$, contradicting Lemma 6.4(i).

So assume that $|[\widetilde{u}, \widetilde{\Sigma}]|=4$. Then, by Proposition $2.12(\mathrm{v}), C_{\widetilde{E} / \widetilde{\Sigma}}(\widetilde{u} \widetilde{\Sigma})$ has orbits of length 1,6 and 9 on $C_{\widetilde{\Sigma}}(\widetilde{u}) /[\widetilde{\Sigma}, \widetilde{u}]$. Hence there are exactly three conjugacy classes of involutions in $\widetilde{\Sigma u}$, two of which have representatives centralized by an element of order three. Assume that $\widetilde{u}$ is one of these. Let $\hat{u}$ be the involution, which is centralized by $S_{1}$, a preimage of a Sylow 3-subgroup of $C_{\widetilde{K}_{1} / \Sigma}(\widetilde{u} \widetilde{\Sigma})$. Set $S_{2}=C_{S_{1}}(\widetilde{u})$. Then, using Lemmas 6.3, 6.2 and 6.4(iv), we see that $\left|S_{2}\right|=3$. Therefore $\tilde{u}$ is not conjugate to $\hat{u}$. In particular, $\widetilde{u}=\hat{u} \widetilde{s}$ where $\widetilde{s} \in C_{\widetilde{\Sigma}}(\widetilde{u}) \backslash[\widetilde{\Sigma}, \widetilde{u}]$. Hence $C_{C_{\widetilde{\Sigma}}(\widetilde{u}) /[\widetilde{\Sigma}, \tilde{u}]}\left(S_{2}\right) \neq 1$. By Proposition 2.12(vi), we get $\left|C_{\widetilde{\Sigma}}\left(S_{2}\right)\right|=4$. So, by Proposition 2.12(iii), $S_{2}$ does not centralize involutions in $\Sigma$. Thus we may assume that $S_{2}=Z$. This contradicts Lemma 6.4(iii), and so we conclude that $C_{\widetilde{E}}(\widetilde{u})$ is a 2-group. Hence (i)(a) and (i)(b) hold.

LeMma 6.6. $\widetilde{y}^{\widetilde{K}} \cap \widetilde{E} \subseteq \widetilde{\Sigma}$.

Proof. Assume that $\widetilde{y}$ is $\widetilde{K}$-conjugate to $\widetilde{u}$ for some involution $\widetilde{u} \in \widetilde{E} \backslash \widetilde{\Sigma}$. By Lemma $6.1,\left|C_{\widetilde{K}}(\widetilde{y})\right|_{2}=2^{12}$ if $\widetilde{E} / \widetilde{\Sigma} \cong \mathrm{SU}_{4}(2)$ or $2^{14}$ if $\widetilde{E} / \widetilde{\Sigma} \cong \mathrm{Sp}_{6}(2)$. This conflicts with information given in Lemma 6.5 . Hence no such conjugation is possible. 
Lemma 6.7. $\widetilde{\Sigma}$ is weakly closed in $\widetilde{K}_{1}$. In particular, $\widetilde{K}_{1}$ contains a Sylow 2-subgroup of $\widetilde{K}$.

Proof. Assume that $T \in \operatorname{Syl}_{2}\left(\widetilde{K}_{1}\right), w \in \widetilde{K}$ and $\widetilde{\Sigma}^{w} \leq T$ with $\widetilde{\Sigma} \neq \widetilde{\Sigma}^{w}$. Then $\widetilde{\Sigma}^{w} \cap \widetilde{E}$ has order at least $2^{7}$ and therefore is generated by conjugates of $\tilde{y}$. Thus Lemma 6.6 implies that $\widetilde{\Sigma}^{w} \cap \widetilde{E} \leq \widetilde{\Sigma}$. But then $\left|\widetilde{\Sigma^{w}} \widetilde{\Sigma} / \widetilde{\Sigma}\right|=2$ and $\left|\widetilde{\Sigma} \cap \widetilde{\Sigma}^{w}\right|=2^{7}$. Since $\widetilde{K}_{1}$ does not contain transvections, we have a contradiction. Hence $N_{\widetilde{K}}(T) \leq \widetilde{K_{1}}$ and, in particular, $T \in \operatorname{Syl}_{2}(\widetilde{K})$.

Lemma 6.8. No element of $\widetilde{\Sigma}$ is $\widetilde{K}$-conjugate to an involution $\widetilde{u} \in \widetilde{K}_{1}$ with $|[\widetilde{\Sigma}, \widetilde{u}]|=4$.

Proof. Assume that the statement is false. Then, by Lemma 6.6, $\widetilde{u}$ is $\widetilde{K}$-conjugate to $\widetilde{x}$. Let $T_{1}$ be a Sylow 2-subgroup of $C_{\widetilde{K}_{1}}(\widetilde{u})$ and $T_{2}$ be a Sylow 2-subgroup of $C_{\widetilde{K}}(\widetilde{u})$ with $T_{1} \leq T_{2}$. By Lemmas 2.12(ii), 6.5 and 6.7, $\left|T_{2}: T_{1}\right|=4$. Let $\widetilde{\Sigma}_{u}$ be the $\widetilde{K}$ conjugate of $\widetilde{\Sigma}$ in $T_{2}$. Then $\left|\widetilde{\Sigma}_{u} \cap T_{1}\right| \geq 2^{6}$. As every subgroup of $\widetilde{\Sigma}$ of order at least $2^{6}$

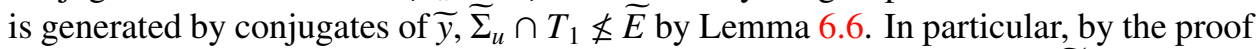
of Lemma $6.5, J>J_{0}$. Therefore, we may suppose that there is a $\widetilde{w} \in \widetilde{\Sigma}_{u} \cap T_{1}$ such that $\widetilde{w}$ inverts $\sigma$. Notice that $\left(\widetilde{\Sigma}_{u} \cap T_{1}\right) \widetilde{\Sigma}$ is normal in $T_{1} \widetilde{\Sigma} \in \operatorname{Syl}_{2}\left(\widetilde{K}_{1}\right)$. In particular, if $\left|\left(\widetilde{\Sigma}_{u} \cap T_{1}\right) \widetilde{\Sigma} / \widetilde{\Sigma}\right|=2^{2}$, then $\widetilde{w} \widetilde{\Sigma}$ is centralized by a maximal subgroup of $T_{1} \widetilde{\Sigma} / \widetilde{\Sigma}$, which is impossible. Hence

$$
\left|\left(\widetilde{\Sigma}_{u} \cap T_{1}\right) \widetilde{\Sigma} / \widetilde{\Sigma}\right| \geq 2^{3} .
$$

In particular, $\left|\left(\widetilde{\Sigma}_{u} \cap T_{1} \cap \widetilde{E}\right) \widetilde{\Sigma} / \widetilde{\Sigma}\right| \geq 2^{2}$ and by Lemma 6.6 all the nontrivial elements of $\left(\widetilde{\Sigma}_{u} \cap T_{1} \cap \widetilde{E}\right) \widetilde{\Sigma} / \widetilde{\Sigma}$ are unitary transvections. This, however, contradicts Proposition 2.12(viii) and proves the lemma.

Lemma 6.9. $\widetilde{y}^{\widetilde{K}} \cap \widetilde{K}_{1} \subseteq \widetilde{\Sigma}$. In particular, $\widetilde{\Sigma}$ is strongly closed in $\widetilde{E}$.

Proof. Suppose that $\widetilde{u} \in \widetilde{y}^{K} \cap \widetilde{K}_{1} \backslash \widetilde{\Sigma}$. Then by Lemmas 6.6 and $6.5, J>J_{0}$ and $\widetilde{u}$ inverts $\sigma$. Furthermore, all involutions in $\widetilde{\Sigma u}$ are conjugate. Hence, for $T_{1} \in$ $\operatorname{Syl}_{2}\left(C_{\widetilde{K}_{1}}(\widetilde{u})\right)$, we obtain $\left|T_{1}\right|=2^{9}$ using Lemma 5.16. Let $T_{2}$ be a Sylow 2-subgroup of $C_{\widetilde{K}}(\widetilde{u})$ with $T_{1} \leq T_{2}$ and $\widetilde{\Sigma}_{u} \leq T_{2}$ be a $\widetilde{K}$-conjugate of $\widetilde{\Sigma}$ in $T_{2}$. By Lemma 6.8, $\left(\widetilde{\Sigma}_{u} \cap T_{1}\right) \backslash \widetilde{\Sigma}$ does not contain elements $v$ with $|[v, \widetilde{\Sigma}]|=4$. So, by Lemma 6.5, $\left(\widetilde{\Sigma}_{u} \cap\right.$ $\left.T_{1}\right) \widetilde{\Sigma} / \widetilde{\Sigma}$ inverts $\sigma \widetilde{\Sigma}$, and this yields $\widetilde{\Sigma}_{u} \cap T_{1} \subseteq\langle\widetilde{u}\rangle \widetilde{\Sigma}$. Since $\left|\widetilde{\Sigma}_{u} \cap T_{1}\right| \geq 2^{5}, \widetilde{\Sigma}_{u} \cap T_{1}=$ $\langle\widetilde{u}\rangle C_{\widetilde{\Sigma}}(\widetilde{u})$ has order $2^{5}$. Hence $T_{2}=T_{1} \widetilde{\Sigma}_{u}$ and $T_{2} / \widetilde{\Sigma}_{u} \cong T_{1} /\langle\widetilde{u}\rangle C_{\widetilde{\Sigma}}(\widetilde{u}) \cong 2 \times \operatorname{Dih}(8)$. But $T_{2} / \widetilde{\Sigma}_{u} \cong \operatorname{SDih}(16)$ by Proposition 2.12(ii), and thus we have a contradiction. Hence $\widetilde{y}^{\bar{K}} \cap \widetilde{K}_{1} \subseteq \widetilde{\Sigma}$.

Lemma 6.10. $\widetilde{\Sigma}$ is strongly closed in $\widetilde{K}_{1}$.

Proof. Assume by way of contradiction that there is some involution $\widetilde{u} \in \widetilde{K}_{1} \backslash \widetilde{\Sigma}$, which is conjugate in $\widetilde{K}$ to some element in $\widetilde{\Sigma}$. By Lemma $6.9, \widetilde{u}$ is $\widetilde{K}$-conjugate to $\widetilde{x}$. By Lemmas 6.8 and $6.5, J>J_{0}$ and we may assume that $\widetilde{u}$ inverts $\sigma$. Furthermore,

$$
C_{\widetilde{E} / \widetilde{\Sigma}}(\widetilde{u}) \cong 2 \times \operatorname{Sym}(4) .
$$


Let $T_{1}$ be a Sylow 2-subgroup of $C_{\widetilde{K}_{1}}(\widetilde{u})$ and $T_{2}$ be a Sylow 2-subgroup of $C_{\widetilde{K}}(\widetilde{u})$, which contains $T_{1}$. Further, let $\widetilde{\Sigma}_{u}$ be the normal subgroup of $T_{2}$ which is $\widetilde{K}$-conjugate to $\widetilde{\Sigma}$. Since, by Proposition 2.12(ix), $C_{\widetilde{\Sigma}}(\widetilde{u})$ is generated by conjugates of $\widetilde{y}$, we have $C_{\widetilde{\Sigma}}(\widetilde{u}) \leq \widetilde{\Sigma}_{u}$ by Lemma 6.9. Since $\left(\widetilde{\Sigma}_{u} \cap T_{1}\right) \widetilde{\Sigma} / \widetilde{\Sigma}=\langle\widetilde{u}\rangle \widetilde{\Sigma} / \widetilde{\Sigma}$,

$$
T_{3}=\widetilde{\Sigma}_{u} \cap T_{1}=C_{\widetilde{\Sigma}}(\widetilde{u})\langle\widetilde{u}\rangle .
$$

Therefore $T_{3}$ is normalized but not centralized by $\widetilde{\Sigma}$ and is centralized by $\widetilde{\Sigma}_{u} \cdot \widetilde{\Sigma}$ and $\widetilde{\Sigma}_{u}$ are contained in $N_{\widetilde{K}}\left(T_{3}\right)$. Let $S_{\widetilde{\Sigma}}$ and $S_{\widetilde{\Sigma_{u}}}$ be Sylow 2-subgroups of $N_{\widetilde{K}}\left(T_{3}\right)$ which contain $\widetilde{\Sigma}$ and $\widetilde{\Sigma}_{u}$, respectively. As, by Lemma 6.7, $\widetilde{\Sigma}$ is weakly closed in $S_{\widetilde{\Sigma}}$ and $\widetilde{\Sigma}_{u}$ is weakly closed in $S_{\widetilde{\Sigma_{u}}}$, we see that $\widetilde{\Sigma}$ and $\widetilde{\Sigma}_{u}$ are conjugate in $N_{\widetilde{K}}\left(T_{3}\right)$. But this is impossible as only one of these subgroups centralizes $T_{3}$.

THEOREM 6.11. $K=K_{1}$.

Proof. Let $T \in \operatorname{Syl}_{2}(K)$. By Lemmas 6.7 and $6.10, \widetilde{\Sigma}$ is strongly closed in $\widetilde{T}$ with respect to $\widetilde{K}$. Hence an application of [5] yields that $\widetilde{L}=\langle\widetilde{\Sigma} \widetilde{K}\rangle$ is an extension of a group of odd order by a product of a 2-group and a number of Bender groups. Furthermore, $\widetilde{\Sigma}$ is the set of involutions in some Sylow 2-subgroup of $T \cap \widetilde{L}$. By Lemma 5.4, $K_{1}$ contains a Sylow 3-subgroup of $K$. Because $C_{K}(\widetilde{\Sigma})=\widetilde{\Sigma}$, we get that $O_{2^{\prime}}(\widetilde{L})=O_{3^{\prime}}(\widetilde{L})$. As $J_{1}$ normalizes $O_{2^{\prime}}(\widetilde{L})$, we get $O_{2^{\prime}}(\widetilde{L})=1$ from Lemma 5.13. Since $\widetilde{K}_{1}$ acts primitively on $\widetilde{\Sigma}$, either $\widetilde{L}=\widetilde{\Sigma}$ and we are done, or $\widetilde{L}$ is a simple group. So suppose that $\widetilde{L}$ is a simple group. Then $N_{\widetilde{L}}(\widetilde{\Sigma})$ acts transitively on $\widetilde{\Sigma}$, which is not possible as $\Sigma$ is extraspecial. This proves that $\widetilde{L}=\widetilde{\Sigma}$ and so $K=K_{1}$.

\section{Proof of Theorem 1.2}

We retain the notation established in previous sections. If $N_{M}(S) / S \cong \operatorname{Dih}(8)$, Theorem 1.2 follows from Theorem 4.7. So we may assume that $N_{M}(S) / S \cong 2 \times$ $\operatorname{Dih}(8)$. Using Theorem 6.11 and Lemma 5.17, we get that $\left.K / \Sigma \cong \mathrm{Aut}_{(} \mathrm{SU}_{4}(2)\right)$, $\left(3 \times \mathrm{SU}_{4}(2)\right): 2$ or $\mathrm{Sp}_{6}(2)$.

Suppose that $K / \Sigma \cong \mathrm{Sp}_{6}(2)$. Then [23] implies that $G \cong \mathrm{Co}_{2}$ and consequently $M=N_{G}(Z)$ has order $2^{8} \cdot 3^{6} \cdot 5$ and shape $3_{+}^{1+4} \cdot 2_{-}^{1+4} \cdot \operatorname{Sym}(5)$, which is not similar to a centralizer of type $\mathrm{PSU}_{6}(2)$ or $\mathrm{F}_{4}(2)$. This contradicts our initial hypothesis. So suppose that $K / \Sigma \cong \operatorname{Aut}\left(\mathrm{SU}_{4}(2)\right)$ or $\left(3 \times \mathrm{SU}_{4}(2)\right): 2$. Then Lemma 2.13 shows that $G$ possesses a subgroup $G_{0}$ of index two. In particular, $C_{G_{0}}(r) / \Sigma \cong \mathrm{SU}_{4}(2)$ or $3 \times \mathrm{SU}_{4}(2)$. Now we see that $N_{G_{0} \cap M}(S) / S \cong \operatorname{Dih}(8)$. Hence Theorem 4.7 gives $G_{0} \cong \mathrm{PSU}_{6}(2)$ or $\mathrm{PSU}_{6}(2): 3$ and so Theorem 1.2 is proved.

\section{Acknowledgements}

The initial draft of this paper was prepared during a visit to the Mathematisches Forschungsinstitut Oberwolfach as part of the Research in Pairs Programme from 30 November to 12 December 2009. The authors are pleased to thank the MFO and its 
staff for the pleasant and stimulating research environment that they provided. The first author is also grateful to the DFG for their support and the mathematics department in Halle for their hospitality.

\section{References}

[1] M. Aschbacher, Finite Group Theory, Cambridge Studies in Advanced Mathematics, 10 (Cambridge University Press, Cambridge, 1986).

[2] R. Brauer and M. Suzuki, 'On finite groups of even order whose 2-Sylow group is a quaternion group', Proc. Natl Acad. Sci. USA 45 (1959), 1757-1759.

[3] J. H. Conway, R. T. Curtis, S. P. Norton, R. A. Parker and R. A. Wilson, Atlas of Finite Groups: Maximal Subgroups and Ordinary Characters for Simple Groups (Clarendon Press, Oxford, 1985).

[4] W. Feit and J. G. Thompson, 'Finite groups which contain a self-centralizing subgroup of order three', Nagoya Math. J. 21 (1962), 185-197.

[5] D. Goldschmidt, '2-fusion in finite groups', Ann. of Math. 99 (1974), 70-117.

[6] D. Gorenstein, Finite Groups (Harper \& Row, New York, 1968).

[7] D. Gorenstein, R. Lyons and R. Solomon, The Classification of the Finite Simple Groups, No. 2, Mathematical Surveys and Monographs, 40.2 (American Mathematical Society, Providence, RI, 1996).

[8] D. Gorenstein, R. Lyons and R. Solomon, The Classification of the Finite Simple Groups, No. 3, Mathematical Surveys and Monographs, 40.3 (American Mathematical Society, Providence, RI, 1998).

[9] J. L. Hayden, 'A characterization of the finite simple group $\mathrm{PSp}_{4}(3)$ ', Canad. J. Math. 25 (1973), 539-553.

[10] B. Huppert, Endliche Gruppen (Springer, Berlin, 1967).

[11] I. M. Isaacs, Character Theory of Finite Groups (AMS Chelsea Publishing, Providence, RI, 2006).

[12] U. Meierfrankenfeld, B. Stellmacher and G. Stroth, 'The structure theorem for finite groups with a large $p$-subgroup', Preprint, 2011.

[13] C. Parker, 'A 3-local characterization of $\mathrm{U}_{6}(2)$ and $\mathrm{Fi}_{22}$ ', J. Algebra 300(2) (2006), 707-728.

[14] C. Parker and P. Rowley, 'A 3-local identification of the alternating group of degree eight, the McLaughlin simple group and their automorphism groups', J. Algebra 319 (2008), 1752-1775.

[15] C. Parker and P. Rowley, 'A 3-local characterization of $\mathrm{Co}_{2}$ ', J. Algebra 323(3) (2010), 601-621.

[16] C. Parker and G. Stroth, 'Strongly p-embedded subgroups', Pure Appl. Math. Q. 7(3 special issue: in honor of Jacques Tits) (2011), 797-858.

[17] C. Parker and G. Stroth, 'Groups which are almost groups of Lie type in characteristic p', Preprint, arXiv: 1110.1308.

[18] C. Parker and G. Stroth, ' $\mathrm{F}_{4}(2)$ and its automorphism group', Preprint, arXiv:1108.1661.

[19] C. Parker, M. R. Salarian and G. Stroth, 'A characterisation of ${ }^{2} E_{6}(2), M(22)$ and $A u t(M(22))$ from a characteristic 3 perspective', Preprint, arXiv:1108.1894.

[20] C. Parker and G. Stroth, 'An identification theorem for the sporadic simple groups $F_{2}$ and $M(23)$ ', Preprint, arXiv:1201.3229v1.

[21] A. R. Prince, 'A characterization of the simple groups $\operatorname{PSp}(4,3)$ and $\operatorname{PSp}(6,2)$ ', J. Algebra 45(2) (1977), 306-320.

[22] A. Seidel, Gruppen lokaler Charakteristik - eine Kennzeichnung von Gruppen vom Lie Typ in ungerader Charakteristik, Dissertation, Universität Halle-Wittenberg, 2009.

http://digital.bibliothek.uni-halle.de/hs/content/titleinfo/397705.

[23] F. Smith, 'A characterization of the .2 Conway simple group', J. Algebra 31 (1974), 91-116.

[24] T. Yoshida, 'Character-theoretic transfer', J. Algebra 52 (1978), 1-38. 
CHRIS PARKER, School of Mathematics,

University of Birmingham, Edgbaston, Birmingham B15 2TT, UK

e-mail: c.w.parker@bham.ac.uk

GERNOT STROTH, Institut für Mathematik,

Universität Halle-Wittenberg, Theodor-Lieser-Str. 5, 06099 Halle, Germany

e-mail: gernot.stroth@mathematik.uni-halle.de 